B O N N E V I L L E P O W E R A D M I N I S T R A T I O Fall Chinook Salmon Spawning Ground Surveys in the Snake River Basin upriver of Lower Granite Dam

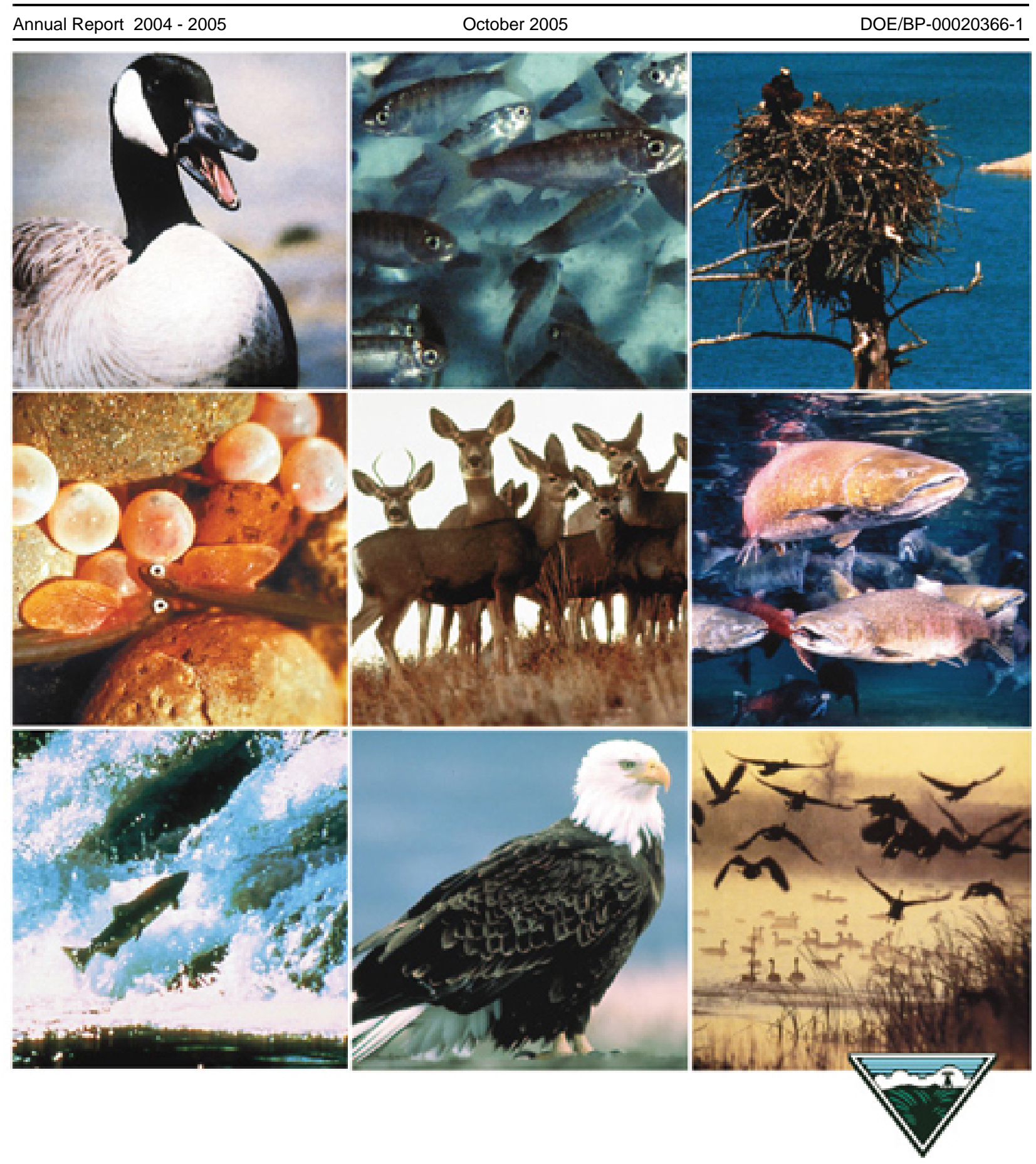


This Document should be cited as follows:

Garcia, A., S. Bradbury, B. Arnsberg, S. Rocklage, P. Groves, "Fall Chinook Salmon Spawning Ground Surveys in the Snake River Basin upriver of Lower Granite Dam", 2004-2005 Annual Report, Project No. 199801003, 60 electronic pages, (BPA Report DOE/BP-00020366-1)

\author{
Bonneville Power Administration \\ P.O. Box 3621 \\ Portland, OR 97208
}

This report was funded by the Bonneville Power Administration (BPA), U.S. Department of Energy, as part of BPA's program to protect, mitigate, and enhance fish and wildlife affected by the development and operation of hydroelectric facilities on the Columbia River and its tributaries. The views in this report are the author's and do not necessarily represent the views of BPA. 


\title{
Fall Chinook Salmon Spawning Ground Surveys in the Snake River Basin Upriver of Lower Granite Dam, 2004
}

\author{
Annual Report \\ November 2004 - October 2005 \\ Prepared by: \\ A. P. Garcia, and S. Bradbury \\ U.S. Fish and Wildlife Service, Idaho Fishery Resource Office \\ Ahsahka, Idaho \\ And \\ B. D. Arnsberg, and S. J. Rocklage \\ Nez Perce Tribe, Fisheries Department \\ Orofino, Idaho \\ And \\ P. A. Groves \\ Idaho Power Company, Environmental Affairs Department \\ Boise, Idaho \\ Prepared for: \\ U.S. Department of Energy \\ Bonneville Power Administration \\ Division of Fish and Wildlife \\ P.O. Box 3621 \\ Portland, OR 97208-3621 \\ Project Number: 1998-010-03 \\ Contract Number: 00020366
}

October 2005 


\section{Introduction}

Redd counts were used to document the spawning distribution of fall Chinook salmon (Oncorhynchus tshawytscha) in the Snake River basin upriver of Lower Granite Dam. The first reported redd counts were from aerial searches conducted intermittently between 1959 and 1978 (Irving and Bjornn 1981, Witty 1988; Groves and Chandler 1996)(Appendix 1). In 1986, the Washington Department of Fish and Wildlife began an annual monitoring program that, in addition to the Snake River, included aerial searches of the Grande Ronde River the first year (Seidel and Bugert 1987), and the Imnaha River in subsequent years (Seidel et al. 1988; Bugert et al. 1989-1991; Mendel et al. 1992). The U. S. Fish and Wildlife Service and Idaho Power Company began contributing to this effort in 1991 by increasing the number of aerial searches conducted each year and adding underwater searches in areas of the Snake River that were too deep to be searched from the air (Connor et al. 1993; Garcia et al. 1994a, 1994b, 1996-2004; Groves 1993; Groves and Chandler 1996). The Nez Perce Tribe added aerial searches in the Clearwater River basin beginning in 1988 (Arnsberg et. al 1992), and the Salmon River beginning in 1992. Currently searches are conducted cooperatively by the Nez Perce Tribe, Idaho Power Company, and U. S. Fish and Wildlife Service.

Our objective for this report was to consolidate the findings from annual redd searches into a single document, containing detailed information about the searches from the most recent spawning season, and summary information from previous years. The work conducted in 2004 was funded by the Bonneville Power Administration, Idaho Power Company, and Bureau of Land Management.

\section{Study Area}

The study area included the free-flowing Snake River between Lower Granite and Hells Canyon dams and portions of the major tributaries that enter therein (Figure 1). We refer to locations using river miles (RM) and river kilometers (RK) based on navigation charts of the Snake River (USACE 1990) and U.S. 
Geological Survey topographical maps. In 2004, searches focused on eight river reaches: (1) the Snake River from the head of Lower Granite Reservoir (RM 147; RK 237) to Hells Canyon Dam (RM 247; RK 397), (2) the Clearwater River to the North Fork Clearwater River (RM 41; RK 66), (3) the North Fork Clearwater River to Dworshak Dam (RM 2; RK 3), (4) the Clearwater River to the South Fork and Middle Fork Clearwater river confluence (RM 74; RK 119); (5) the South Fork Clearwater River to Butcher Creek (RM 12; RK 19), (6) the Grande Ronde River to Wildcat Creek (RM 53; RK 85), (7) the Salmon River to French Creek (RM 105; RK 169), and (8) the Imnaha River to the Cow Creek Bridge (RM 4; RK 6). The number of searches per reach varied between reaches and years. Searches were conducted in other portions of the aforementioned Snake River tributaries, and in the Potlatch River and Asotin Creek, though less consistently.

\section{Methods}

Redd searches were conducted from a helicopter flown at an altitude of about $700-\mathrm{ft}$. or less. At minimum, observations were made by a primary observer and the pilot, though typically at least one additional observer was present. Redd locations were determined by referencing navigation charts of the Snake River (USACE 1990), or U. S. Geological Survey topographical maps, or using the Global Positioning System (GPS) coupled with mapping software. From 1991 to 2004, searches in the Snake, Clearwater, Grande Ronde, and Imnaha rivers were scheduled to be conducted at 7-d intervals starting around mid-October and ending around mid-December. In previous years, and in the other rivers, searches were generally conducted less frequently. In most years some scheduled searches were canceled or shortened due to poor visibility or inclement weather. Redds observed in the Snake River that could not clearly be distinguished from the air were examined from the ground (hereafter referred to as ground truthing) beginning in 1991. Ground truthing was also performed in the other rivers though less consistently. Only the number of new redds observed on each search were recorded.

Redd searches in the Snake River were also conducted using underwater search methods in areas too 
deep to be effectively searched from the air. In 1991 and 1992, deep-water redd searches were conducted using methods developed by Swan (1989) that involved direct observation of bottom substrates by scuba divers (Connor et al. 1993; Garcia et al. 1994a). From 1993-2004, deep-water redd searches were conducted using a submersible video camera connected to a 65 -ft camera cable, viewing monitor(s), and a video-recording device. The search camera was either enclosed in an aluminum sheath mounted on a 90-lb lead weight, or attached to an aluminum frame mounted between two 30-lb lead weights, and could be adjusted 45 to 90 degrees down from horizontal (Groves and Garcia, 1998). The camera was suspended from a boat using a wire rope passed through a roller on the bow, and attached to a sounding-reel/depth-indicator mounted in the boat cabin.

Underwater searches were accomplished by passing the camera over the river bottom in parallel paths spaced roughly $30 \mathrm{ft}$. apart. Distance between paths was determined by sight estimation, measuring cord laid along the shore, or GPS. The distance between the camera and river bottom, and the angle of the camera, was adjusted to maximize the amount of viewable area without losing the ability to observe details of the bottom substrates. If a redd was observed, the distance between passes in the search pattern was reduced, and in most cases, the entire area was searched at least two times in the course of the spawning season.

Camera location was recorded using electronic surveying equipment or GPS. Searches were video taped when large groups of redds were found, and the numbers of redds were verified by reviewing recorded searches while referencing plots of redd coordinates. In areas where redds overlapped and could not be identified individually, the perimeter of the redd group (or patch) was surveyed and the overall area divided by $492.5 \mathrm{ft}^{2}$-per-redd (45.8- $\mathrm{m}^{2}$-per-redd) based on measurements of fall Chinook salmon redds in the Snake and Columbia rivers (Groves and Chandler 2001). This produced an index count of the total number of redds in the group.

Underwater searches were limited to areas greater than about 10-ft deep with a dominant bottom 
substrate particle size (Bovee 1982) ranging from 1- to 6-in. diameter (Raleigh et al. 1986). In 1991 and 1992, a few pilot searches were conducted at known spawning sites. From 1993 to 2002, search crews attempted to annually search about 90 areas that fit the substrate size and the depth criteria (based on Hells Canyon Dam discharged of about 9,000 cfs). In 2003 and 2004, search crews attempted to search all deep-water sites where redds were observed in previous years. However, additional searches (of new and old sites) were conducted whenever possible.

\section{Results and Discussion}

\section{Snake River}

In 2004, a total of 1,709 redds were observed in the Snake River (Table 1), of which 1,218 were observed during eight aerial searches (Table 2) and 491 during searches of 67 deep-water sites (Tables 3 and 4). Visibility ratings were reported as either "fair" or "good" during aerial searches (Table 5). The locations of all redds counted in the Snake River study area during aerial searches since 1986 are given in Appendix

2. The numbers of searches conducted in the Snake and other rivers are given in Table 6. The locations of all redds observed using submersible cameras are given in Table 7. The locations of all redds counted (aerial and underwater counts combined) in the Snake River study area since 1986 are given in Appendix 3. Redds counted in the Snake River amounted to roughly $67 \%$ of all redds observed upriver of Lower Granite Dam in 2004 , compared to $68 \%$ in $2003,60 \%$ in $2002,54 \%$ in $2001,65 \%$ in $2000,64 \%$ in 1999 , $61 \%$ in 1998, and 31\% in 1997 . The percentage of redds counted using submersible cameras from 1993 to 2004 averaged $28.5 \% \pm 9.6 \%$ for the Snake River, and $16.8 \% \pm 6.3 \%$ for all redds counted above Lower Granite Dam. In 2004, the greatest number of new redds were observed on November 8 (Table 2).

\section{Clearwater River basin}

A total of 631 redds were observed in the Clearwater River basin in 2004, 630 in the Clearwater and North 
Fork Clearwater rivers (Tables 1 and 9) during 10 aerial searches for fall Chinook salmon redds (Table 6), and one in the Potlatch River during a ground search (on 2-Nov) for coho salmon redds (Table 1; Scott Everett, NPT, personal communication). Observation conditions were poor on each flight over the Clearwater River in 2004 (Table 9). The locations of all redds counted in the Clearwater River are given in Appendix 4. Redds counted in the Clearwater River basin amounted to $25 \%$ of all redds observed upriver of Lower Granite Dam in 2004, compared to $26 \%$ in $2003,28 \%$ in $2002,26 \%$ in $2001,32 \%$ in $2000,32 \%$ in 1999 , and $26 \%$ in 1998. In 2004, the greatest numbers of new redds (RM 0-45) were observed on October 27 (Table 8).

\section{Asotin Creek}

A total of four redds were observed during two searches of Asotin Creek in 2004 (Tables 1 and 6). Asotin Creek was surveyed for fall Chinook salmon redds from 1989-1992, but no redds were found (Table 1). In 2003, redds were observed incidentally (Garcia et al. 2004). Searches in 2004 were conducted on November 15 and 29, and covered from the creek mouth to RM 13. Search conditions were reported as "Good" on the first flight, and "Fair" on the second. All four redds were observed on the first flight, one at RM 0.05, and three at RM 1.0.

\section{Grande Ronde River}

A total of 162 redds were observed during eight searches of the Grande Ronde River in 2004 (Tables 1, 6, and 12). Observation conditions varied from "Fair" to "Excellent" (Table 11). Redds counted in the Grande Ronde River amounted to $6.3 \%$ of all redds observed upriver of Lower Granite Dam in 2004, compared to $4 \%$ in $2003,6 \%$ in $2002,15 \%$ in $2001,1 \%$ in $2000,2 \%$ in $1999,8 \%$ in 1998 , and $29 \%$ in 1997. The locations of all redds counted in the Grande Ronde River are given in Appendix 5. In 2004, the greatest numbers of new redds were observed on November 10 (Table 10). 


\section{Salmon River}

A total of 21 redds were observed during three searches of the Salmon River in 2004 (Tables 1, 6, and 12). River discharge at RM 53.7 ranged from 3,650-4,680, and observation conditions were reported as "Fair". Redds counted in the Salmon River amounted to about $1 \%$ of all redds observed upriver of Lower Granite Dam in 2004, compared to $1 \%$ in $2003,2 \%$ in 2001 and $2002,0 \%$ in $2000,0 \%$ in $1999,1 \%$ in 1998, and 1\% in 1997. The locations of all redds counted in the Salmon River are given in Appendix 6.

\section{Imnaha River}

A total of 35 redds were observed during eight searches of the Imnaha River in 2004 (Tables 1, 6, and 13). Observation conditions were reported as "Good" to "Excellent" (Table 14). Redds counted in the Imnaha River amounted to about 1\% of all redds observed upriver of Lower Granite Dam in 2004, compared to $4 \%$ in 2003 and 2002, 3\% in 2001, 2\% in 2000, 2\% in 1999, 4\% in 1998, and 2\% in 1997. The locations of all redds counted in the Imnaha River are given in Appendix 7. In 2004, the greatest number of new redds were observed on November 10.

\section{Summary}

A total of 2,562 fall Chinook salmon redds were observed upriver of Lower Granite Dam in 2004 (Figure 2). This was the greatest number of redds counted since annual searches began in 1986 . The increase in the number of redds counted corresponded with an increase in the number of adult fall Chinook salmon counted in the Lower Granite Dam fish ladder (Table 15 and Figure 2). The number of adult fish counted

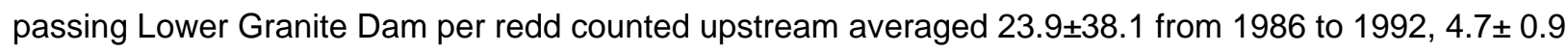
from 1993 to 2004, and was 5.7 in 2004 (Table 15). Most of the redds were observed in the Snake River (67\%) and the Clearwater River basin (25\%). 


\section{References}

Arnsberg, B. D., W. P. Connor, and E. Connor. 1992. Mainstem Clearwater River study: Assessment for salmonid spawning, incubation, and rearing. Final Report by the Nez Perce Tribe, Contract DEAl79-87-BP37474 to Bonneville Power Administration, Portland, Oregon.

Bovee, K. D. 1982. A guide to stream habitat analysis using the Instream Flow Incremental Methodology. Instream Flow Information Paper 12, FWS/OBS-82/26, U.S. Fish and Wildlife Service, Office of Biological Services, Washington, D.C.

Bugert, R., P. Seidel, P. LaRiviere, D. Marbach, S. Martin, and L. Ross. 1989. Lower Snake Compensation Plan, Lyons Ferry Hatchery Evaluation Program, 1988 Annual Report. Cooperative Agreement 14-16-001-88519, U.S. Fish and Wildlife Service, Boise, Idaho.

Bugert, R., P. LaRiviere, D. Marbach, S. Martin, L. Ross, and D. Geist. 1990. Lower Snake Compensation Plan, Lyons Ferry Hatchery Evaluation Program, 1989 Annual Report. Cooperative Agreement 14-16-0001-89525, U.S. Fish and Wildlife Service, Boise, Idaho.

Bugert, R., and six coauthors. 1991. Lyons Ferry Hatchery Evaluation Program, 1990 annual report. Cooperative Agreement 14-16-001-90525 to Lower Snake River Compensation Plan, U.S. Fish and Wildlife Service, Boise, Idaho.

Chapman, D. W., D. E. Weitkamp, T. L. Welsh, M. B. Dell, and T. H. Schadt. 1986. Effects of river flow on the distribution of Chinook salmon redds. Transactions of the American Fisheries Society $115: 537-547$.

Connor, W. P., A. P. Garcia, H. L. Burge, and R. H. Taylor. 1993. Fall Chinook salmon spawning in free- 
flowing reaches of the Snake River. Pages 1-29 in D. W. Rondorf and W. H. Miller, editors. Identification of the spawning, rearing, and migratory requirements of fall Chinook salmon in the Columbia River basin. 1991 Annual Report to Bonneville Power Administration, Contract DEAl79-91BP21708, Portland, Oregon.

Garcia, A. P., W.P. Connor, and R.H. Taylor. 1994a. Fall Chinook spawning ground surveys in the Snake River. Pages 1-19 in D.W. Rondorf and W.H. Miller, editors. Identification of the spawning, rearing, and migratory requirements of fall Chinook salmon in the Columbia River basin. 1992 Annual Report to Bonneville Power Administration, Contract DE-Al79-91BP21708, Portland, Oregon.

Garcia, A. P., W.P. Connor, and R.H. Taylor. 1994b. Fall Chinook spawning ground surveys in the Snake River. Pages 1-21 in D.W. Rondorf and K.F. Tiffan, editors. Identification of the spawning, rearing, and migratory requirements of fall Chinook salmon in the Columbia River basin. 1993 Annual Report to Bonneville Power Administration, Contract DE-Al79-91BP21708, Portland, Oregon.

Garcia, A. P., and six coauthors. 1996. Fall Chinook spawning ground surveys in the Snake River, 1994. Pages 1-18 in D.W. Rondorf and K.F. Tiffan, editors. Identification of the spawning, rearing, and migratory requirements of fall Chinook salmon in the Columbia River basin. 1994 Annual Report to Bonneville Power Administration, Contract DE-Al79-91BP21708, Portland, Oregon.

Garcia, A. P., W.P Connor, R.D. Nelle, R.D. Waitt, E.A. Rockhold, and R.S. Bowen. 1997. Fall Chinook spawning ground surveys in the Snake River, 1995. Pages 1-17 in D.W. Rondorf and K.F. Tiffan, editors. Identification of the spawning, rearing, and migratory requirements of fall Chinook salmon in the Columbia River basin. 1995 Annual Report to Bonneville Power Administration, Contract DE-AI79-91BP21708, Portland, Oregon. 
Garcia, A. P., R.D. Waitt, C.A. Larsen, S.M. Bradbury, B.D. Arnsberg, M. Key, P.A. Groves. 1999. Fall Chinook salmon spawning ground surveys in the Snake River basin upriver of Lower Granite Dam, 1998. Pages 7-19 in A.P. Garcia editor. Spawning distribution of fall Chinook salmon in the Snake River. 1998 Annual Report to Bonneville Power Administration, Project number 9801003, Contract 98-Al-37776, Portland, Oregon.

Garcia, A. P., R.D. Waitt, C.A. Larsen, D. Burum, B.D. Arnsberg, M. Key, P.A. Groves. 2000. Fall Chinook salmon spawning ground surveys in the Snake River basin upriver of Lower Granite Dam, 1999. Pages 10-28 in A.P. Garcia editor. Spawning distribution of fall Chinook salmon in the Snake River. 1999 Annual Report to Bonneville Power Administration, Project number 9801003, Contract 98-AI-37776, Portland, Oregon.

Garcia, A. P., R.D. Waitt, C.A. Larsen, D. Burum, B.D. Arnsberg, M. Key, P.A. Groves. 2001. Fall Chinook salmon spawning ground surveys in the Snake River basin upriver of Lower Granite Dam, 2000. Pages 13-31 in A.P. Garcia editor. Spawning distribution of fall Chinook salmon in the Snake River. 2000 Annual Report to Bonneville Power Administration, Project number 199801003, Contract 98-AI-37776, Portland, Oregon.

Garcia, A. P., and six coauthors. 2003. Fall Chinook salmon spawning ground surveys in the Snake River basin upriver of Lower Granite Dam. Pages 14-22 in A.P. Garcia editor. Spawning distribution of fall Chinook salmon in the Snake River. 2001 Annual Report to Bonneville Power Administration, Project number 199801003, Contract 98-Al-37776, Portland, Oregon.

Garcia, A. P., S. Bradbury, B. D. Arnsberg, S. J. Rocklage, and P. A. Groves. 2003. Fall Chinook salmon spawning ground surveys in the Snake River basin upriver of Lower Granite Dam, 2002. 2002 Annual Report to Bonneville Power Administration, Project number 199801003, Contract 98-AI37776, Portland, Oregon. 
Garcia, A. P., S. Bradbury, B. D. Arnsberg, S. J. Rocklage, and P. A. Groves. 2004. Fall Chinook salmon spawning ground surveys in the Snake River basin upriver of Lower Granite Dam, 2003. 2003 Annual Report to Bonneville Power Administration, Project number 199801003, Contract 98-AI37776, Portland, Oregon.

Groves, P. A. 1993. Habitat available for, and used by, fall Chinook salmon within the Hells Canyon Reach of the Snake River. Idaho Power Company, Boise, Idaho.

Groves, P. A, and J.A. Chandler. 1996. A summary of fall Chinook salmon (Oncorhynchus tshawytscha) redd surveys within the Hells Canyon reach of the Snake River, Idaho: 1991-1995. Report to the National Marine Fisheries Service, Silver Springs, Maryland.

Groves, P. A., and J.A. Chandler. 2001. Chapter 3: The quality and availability of fall Chinook salmon spawning and incubation habitat downstream of the Hells Canyon Complex. In: P.A. Groves, editor. Evaluation of anadromous fish potential within the mainstem Snake River, downstream of the Hells Canyon Complex of reservoirs. Technical Appendices for Hells Canyon Complex Hydroelectric Project. Technical Report E.3.1-3. Idaho Power Company, Boise, Idaho.

Groves, P. A., and A.P. Garcia. 1998. Two carriers used to suspend an underwater video camera from a boat. North American Journal of Fisheries Management18:1004-1007.

Irving, J. S. and T.C. Bjornn. 1981. Status of Snake River fall Chinook salmon in relation to the Endangered Species Act. Prepared for the U.S. Fish and Wildlife Service, Portland, Oregon.

Mendel, G. K., and six coauthors. 1992. Lower Snake River Compensation Plan Lyons Ferry fall Chinook salmon hatchery program. 1991 Evaluation Report. Cooperative Agreement 14-160001-91534, Washington Department of Fisheries report to the U.S. Fish and Wildlife Service, 
Lower Snake River Compensation Plan Office, Boise, Idaho.

Raleigh, R. F., W.J. Miller, and P.C. Nelson. 1986. Habitat suitability index models and instream flow suitablility curves: Chinook salmon. U.S. Fish and Wildlife Service, Biological Report 82(10.122).

Seidel, P., and R. Bugert. 1987. Lower Snake River Compensation Plan, Lyons Ferry Salmon Evaluation Program, 1986 Annual Report. Cooperative Agreement 14-16-0001-86521. U.S. Fish and Wildlife Service, Boise, Idaho.

Seidel, P., R. Bugert, and P. LaRiviere, D. Marbach, S. Martin, and L. Ross. 1988. Lower Snake River Compensation Plan, Lyons Ferry Evaluation Program, 1987 Annual Report. Cooperative Agreement 14-16-0001-87512. U.S. Fish and Wildlife Service, Boise, Idaho.

Swan, G.A. 1989. Chinook salmon spawning surveys in deep waters of a large, regulated river. Regulated Rivers: Research and Management 4:355-370.

USACE (U.S. Army Corp of Engineers). 1986-2004. Annual fish passage reports, Columbia and Snake Rivers. North Pacific Division, U.S. Army Corps of Engineers, Portland and Walla Walla Districts.

USACE (U.S. Army Corp of Engineers). 1990. Navigation charts of the Snake River, Oregon, Washington, and Idaho. Lewiston to Johnson Bar. U.S. Army Corps of Engineers, Walla Walla District, Walla Walla, Washington.

Witty, K. L. 1988. Annual Fish Report. Wallowa Fish District. Oregon Department of Fish and Wildlife, Enterprise, Oregon. 


\section{ACKNOWLEDGMENTS}

We thank individuals at the U.S. Bureau of Land Management - Cottonwood Resource Area Office, and U.S. Forest Service - Wallowa Whitman National Forest, for their contributions to this project. We extend a special thanks to our colleagues at U.S. Fish and Wildlife Service - Idaho Fishery Resource Office, the Nez Perce Tribe - Fisheries Department, the Washington Department of Fish and Wildlife - Snake River Laboratory, and the Idaho Power - Environmental Affairs Department, for their assistance. Finally, we thank Debbie Docherty, Project Manager, Bonneville Power Administration for her efforts. 
Figures and Tables 


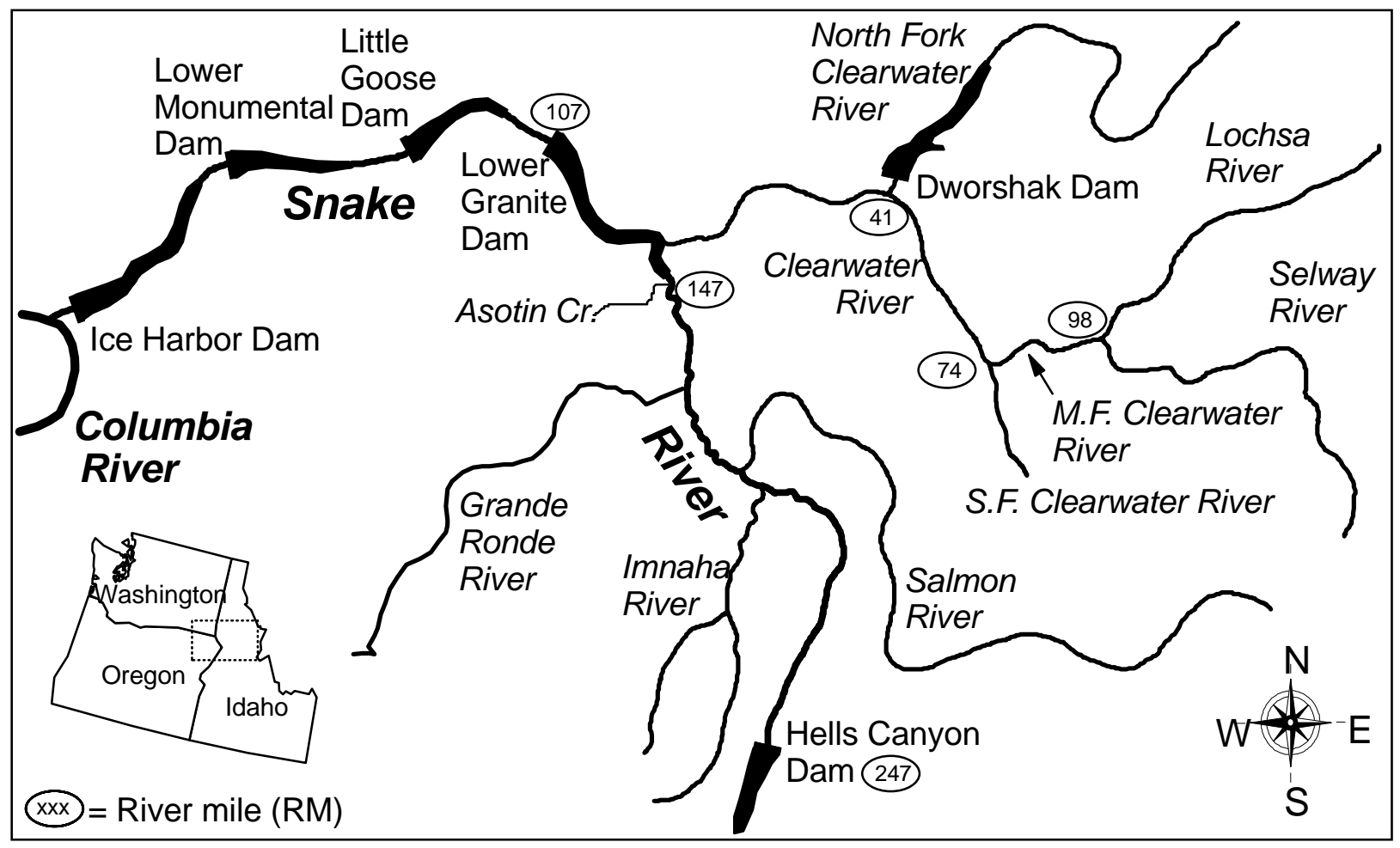

Figure 1. Map of the Snake River drainage in Oregon, Washington, and parts of Idaho. 


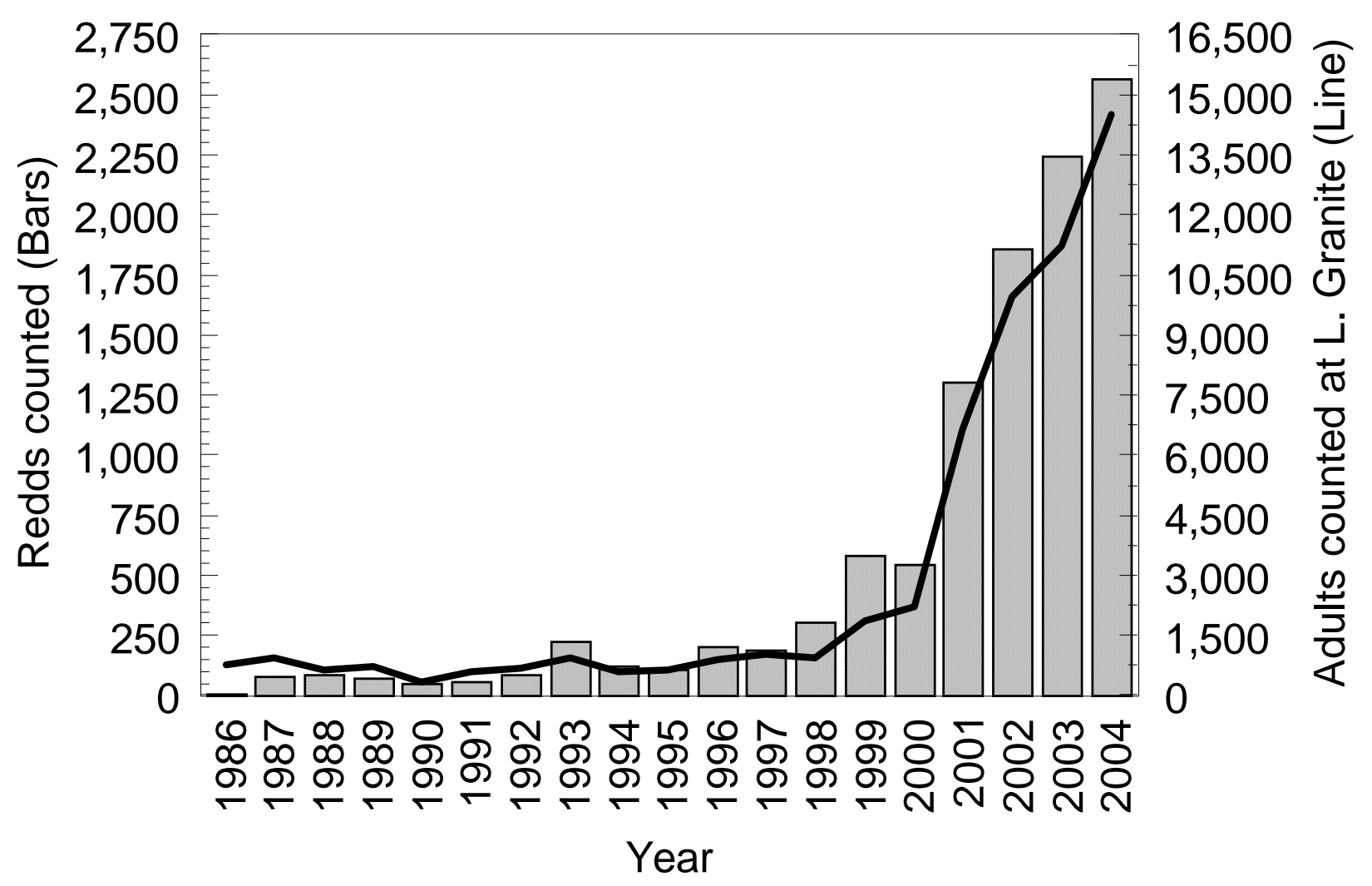

Figure 2. Number of adult fall Chinook salmon counted at Lower Granite Dam, and number of redds counted above the dam, 1986-2004 (Fish counts from USACE 1986-2004, and D. Milks, WDFW, unpublished data). 
Table 1. Number of fall Chinook salmon redds counted in the Snake River and tributaries between Lower Granite and Hells Canyon dams, $1986-2004$. An empty cell indicates no searches were conducted in the corresponding river and year. Some of the data is broken down into method, and river mile (RM) sections. Data collected by the Washington Department of Fish and Wildlife, Nez Perce Tribe, Idaho Power Company, and the U.S. Fish and Wildlife Service. Abbreviations: SR=Snake River, LCR=Lower Clearwater River, NFCR=North Fork Clearwater River, UCR=Upper Clearwater River, SFCR=South Fork Clearwater River, MFCR=Middle Fork Clearwater River, GRR=Grande Ronde River.

\begin{tabular}{|c|c|c|c|c|c|c|c|c|c|c|c|c|c|c|c|c|c|c|c|}
\hline \multirow[b]{2}{*}{ Location } & \multicolumn{19}{|c|}{ Year } \\
\hline & 1986 & 1987 & 1988 & 1989 & 1990 & 1991 & 1992 & 1993 & 1994 & 1995 & 1996 & 1997 & 1998 & 1999 & 2000 & 2001 & 2002 & 2003 & 2004 \\
\hline SR (helicopter) ${ }^{\mathrm{a}}$ & 7 & 66 & 64 & 58 & 37 & 41 & 47 & 60 & 53 & 41 & 71 & 49 & 135 & 273 & 255 & 535 & 878 & 1,118 & 1,218 \\
\hline SR (video) ${ }^{b}$ & & & & & & 5 & 0 & 67 & 14 & 24 & 33 & 9 & 50 & 100 & 91 & 174 & 235 & 394 & 491 \\
\hline LCR (RM0-41) & & & 21 & 10 & 4 & 4 & 25 & 36 & 30 & 20 & 66 & 58 & 78 & 179 & 164 & 290 & 520 & 544 & 592 \\
\hline Potlatch River & & & & & & & & & & & & & & 7 & 0 & 24 & 3 & 1 & 1 \\
\hline $\mathrm{NFCR}^{\mathrm{c}}$ & & & 0 & 0 & 0 & 0 & 0 & 0 & 7 & 0 & 2 & 14 & 0 & 1 & 0 & 1 & 0 & 8 & 2 \\
\hline $\begin{array}{l}\text { UCR } \\
\text { (RM 42-74) }\end{array}$ & & & & & & & 1 & 0 & 0 & 0 & 0 & 0 & 0 & 2 & 8 & 16 & 4 & 19 & 36 \\
\hline SFCR & & & & & & & 0 & 0 & 0 & 0 & 1 & 0 & 0 & 2 & 1 & 5 & 0 & 0 & 0 \\
\hline $\begin{array}{l}\text { MFCR } \\
\text { (RM 75- 98) }\end{array}$ & & & & & & & & & 0 & 0 & 0 & 0 & 0 & 0 & 0 & 0 & 0 & 0 & 0 \\
\hline Selway River & & & & & & & & & 0 & 0 & 0 & 0 & 0 & 0 & 0 & 0 & 0 & 0 & 0 \\
\hline Asotin Creek & & & & 0 & 0 & 0 & 0 & & & & & & & & & & & 3 & 4 \\
\hline GRR & 0 & 7 & 1 & 0 & 1 & 0 & 5 & 49 & 15 & 18 & 20 & 55 & 24 & 13 & 8 & 197 & 111 & 93 & 162 \\
\hline Salmon River & & & & & & & 1 & 3 & 1 & 2 & 1 & 1 & 3 & 0 & 0 & 22 & 31 & 18 & 21 \\
\hline Imnaha River & & 0 & 1 & 1 & 3 & 4 & 3 & 4 & 0 & 4 & 3 & 3 & 13 & 9 & 9 & 38 & 72 & 43 & 35 \\
\hline Totals & 7 & 73 & 87 & 69 & 45 & 54 & 82 & 219 & 120 & 109 & 197 & 189 & 303 & 586 & 536 & 1,302 & 1,854 & 2,241 & 2,562 \\
\hline
\end{tabular}

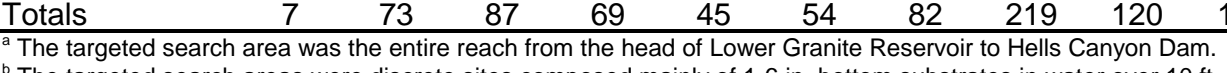

${ }^{\mathrm{b}}$ The targeted search areas were discrete sites composed mainly of $1-6 \mathrm{in}$. bottom substrates in water over $10 \mathrm{ft}$. deep. The number of sites searched varied. Searchers recorded a $170 \mathrm{~m}{ }^{2}$ patch of disturbed substrates in 1995, $238 \mathrm{~m}^{2}$ in 1996, 1,226 $\mathrm{m}^{2}$ in 2001, 1,282 $\mathrm{m}^{2}$ in 2002, 1,122 $\mathrm{m}^{2}$ in 2003, and 1,479 $\mathrm{m}^{2}$ in 2004, at RM 179.6, 1,184 $\mathrm{m}^{2}$ in 2003, and 1,257 $\mathrm{m}^{2}$ in 2004 at RM $212.2,1,259 \mathrm{~m}^{2}$ at RM158.0 in 2004, and $981 \mathrm{~m}^{2}$ at RM193.0 in 2004. In this report we used a conversion factor of $45.8 \mathrm{~m}^{2}$ per redd to estimate the numbers of redds within these patches.

Searches covered from the mouth to the Ahsahka boat ramp in 2002. Searches covered from the mouth to Dworshak Dam in previous years. 
Table 2. New fall Chinook salmon redds counted during aerial searches of the Snake River in 2004 (Data collected by Idaho Power Company and the U.S. Fish and Wildlife Service). Counts are presented by river mile (RM), river kilometer (RK), and date. An empty cell indicates no redds were observed on the corresponding location and date. A dash indicates no search was conducted at the corresponding river mile.

\begin{tabular}{|c|c|c|c|c|c|c|c|c|c|c|}
\hline \multirow[b]{2}{*}{$\mathrm{RM}$} & \multirow[b]{2}{*}{ RK } & \multicolumn{8}{|c|}{ New Redds By Flight Date } & \multirow[b]{2}{*}{ Totals } \\
\hline & & 18 -Oct & 25 -Oct & 1-Nov & 8-Nov & 15-Nov & 22-Nov & 29-Nov & 6-Dec & \\
\hline 148.5 & 238.9 & & & 17 & 14 & 23 & 5 & & & 59 \\
\hline 149.4 & 240.4 & 1 & & 4 & & 4 & & & & 9 \\
\hline 149.6 & 240.7 & & & 2 & & & & & & 2 \\
\hline 151.5 & 243.8 & & & 1 & 1 & 1 & & & & 3 \\
\hline 151.9 & 244.4 & & 10 & 5 & 9 & & 2 & & & 26 \\
\hline 152.3 & 245.1 & & & 7 & 11 & 6 & 11 & 11 & & 46 \\
\hline 152.4 & 245.2 & & & 2 & & & & & & 2 \\
\hline 152.8 & 245.9 & & 1 & & & & & & & 1 \\
\hline 153.2 & 246.5 & & 1 & 1 & & 3 & & & & 5 \\
\hline 155.0 & 249.4 & & & & & 9 & 4 & & & 13 \\
\hline 155.9 & 250.8 & & & & & & & 2 & 9 & 11 \\
\hline 156.4 & 251.6 & & & 3 & 5 & 2 & & & & 10 \\
\hline 157.6 & 253.6 & & & 1 & & & & & & 1 \\
\hline 158.0 & 254.2 & & & & 12 & 3 & 5 & 5 & & 25 \\
\hline 159.7 & 257.0 & & & & & & 1 & & & 1 \\
\hline 161.0 & 259.0 & & 3 & 7 & 3 & 2 & & 1 & & 16 \\
\hline 162.4 & 261.3 & & & 2 & 15 & 5 & 5 & & & 27 \\
\hline 163.7 & 263.4 & & & & & 3 & 2 & & & 5 \\
\hline 164.7 & 265.0 & & 5 & 5 & 10 & 7 & 1 & & & 28 \\
\hline 165.3 & 266.0 & & 5 & 3 & 3 & 6 & 7 & 3 & & 27 \\
\hline 165.8 & 266.8 & & 7 & 20 & 21 & 2 & 6 & 4 & & 60 \\
\hline 166.2 & 267.4 & & & & 1 & & & & & 1 \\
\hline 168.7 & 271.4 & & 4 & 20 & 20 & & & 4 & & 48 \\
\hline 169.7 & 273.0 & & & & 2 & 3 & 1 & & & 6 \\
\hline 171.9 & 276.6 & & & & & & & 1 & & 1 \\
\hline 172.5 & 277.6 & & 1 & 1 & 4 & 3 & 1 & 1 & & 11 \\
\hline 173.9 & 279.8 & & & 2 & 4 & 3 & & & & 9 \\
\hline
\end{tabular}


Table 2 (Continued)

New fall Chinook salmon redds counted during aerial searches of the Snake River in 2004 (Data collected by Idaho Power Company and the U.S. Fish and Wildlife Service). Counts are presented by river mile (RM), river kilometer (RK), and date. An empty cell indicates no redds were observed on the corresponding location and date. A dash indicates no search was conducted at the corresponding river mile.

\begin{tabular}{|c|c|c|c|c|c|c|c|c|c|c|}
\hline \multirow[b]{2}{*}{$\mathrm{RM}$} & \multirow[b]{2}{*}{ RK } & \multicolumn{8}{|c|}{ New Redds By Flight Date } & \multirow[b]{2}{*}{ Totals } \\
\hline & & 18-Oct & $25-$ Oct & 1-Nov & 8-Nov & 15-Nov & 22-Nov & 29-Nov & 6-Dec & \\
\hline 175.9 & 283.0 & & & 1 & 3 & & & & & 4 \\
\hline 176.7 & 284.3 & & & & 3 & 4 & & & & 7 \\
\hline 177.9 & 286.2 & & & & 1 & & & & & 1 \\
\hline 178.3 & 286.9 & & & 2 & 2 & 2 & & 4 & & 10 \\
\hline 178.5 & 287.2 & & & & 1 & & & & & 1 \\
\hline 178.9 & 287.9 & & 3 & 5 & & 2 & & 1 & & 11 \\
\hline 179.5 & 288.8 & & 2 & 3 & & 1 & & & & 6 \\
\hline 179.6 & 289.0 & & & 3 & 7 & 7 & & & & 17 \\
\hline 181.7 & 292.4 & & & 5 & 2 & 3 & & & & 10 \\
\hline 183.2 & 294.8 & & & & & & 1 & & & 1 \\
\hline 183.4 & 295.1 & & & 1 & & 6 & 1 & & & 8 \\
\hline 187.7 & 302.0 & & 1 & 2 & & & & & & 3 \\
\hline 188.2 & 302.8 & & 1 & & & & & & & 1 \\
\hline 190.0 & 305.7 & & & 1 & & & & & & 1 \\
\hline 190.2 & 306.0 & & 1 & & & & & 1 & & 2 \\
\hline 190.8 & 307.0 & & & 3 & 1 & 7 & 3 & 1 & 2 & 17 \\
\hline 191.0 & 307.3 & & & & & & & & 2 & 2 \\
\hline 191.1 & 307.5 & & & 1 & & & & & & 1 \\
\hline 191.7 & 308.4 & & & 2 & & 2 & 2 & & & 6 \\
\hline 193.6 & 311.5 & & & 1 & 2 & & & & & 3 \\
\hline 193.7 & 311.7 & & & 1 & 1 & 7 & & & & 9 \\
\hline 193.8 & 311.8 & & & & 1 & & & & & 1 \\
\hline 194.0 & 312.1 & & 2 & 15 & & 4 & & & & 21 \\
\hline 194.2 & 312.5 & & & & & & 1 & & & 1 \\
\hline 196.0 & 315.4 & & 2 & 18 & 9 & & & & & 29 \\
\hline 198.2 & 318.9 & & 3 & 10 & 1 & 2 & 1 & & & 17 \\
\hline
\end{tabular}


Table 2 (Continued)

New fall Chinook salmon redds counted during aerial searches of the Snake River in 2004 (Data collected by Idaho Power Company and the U.S. Fish and Wildlife Service). Counts are presented by river mile (RM), river kilometer (RK), and date. An empty cell indicates no redds were observed on the corresponding location and date. A dash indicates no search was conducted at the corresponding river mile.

\begin{tabular}{|c|c|c|c|c|c|c|c|c|c|c|}
\hline \multirow[b]{2}{*}{ RM } & \multirow[b]{2}{*}{ RK } & \multicolumn{8}{|c|}{ New Redds By Flight Date } & \multirow[b]{2}{*}{ Totals } \\
\hline & & $18-O c t$ & $25-$ Oct & 1-Nov & 8-Nov & 15-Nov & 22-Nov & 29-Nov & 6-Dec & \\
\hline 198.8 & 319.9 & & 2 & 10 & 9 & 9 & & 1 & & 31 \\
\hline 201.0 & 323.4 & & & 2 & 2 & & & & & 4 \\
\hline 205.3 & 330.3 & & & 2 & 9 & 1 & & & & 12 \\
\hline 205.4 & 330.5 & & & & 5 & & & & & 5 \\
\hline 206.5 & 332.3 & & & 2 & 11 & 2 & & & & 15 \\
\hline 206.6 & 332.4 & & & & 1 & 1 & & & & 2 \\
\hline 207.5 & 333.9 & & & & 1 & & & & & 1 \\
\hline 207.8 & 334.4 & & & & 4 & & & & & 4 \\
\hline 208.0 & 334.7 & & 3 & 28 & 13 & & 1 & 1 & & 46 \\
\hline 211.9 & 340.9 & & & 19 & 4 & 3 & 2 & & & 28 \\
\hline 212.3 & 341.6 & & & & & 3 & 1 & & & 4 \\
\hline 213.6 & 343.7 & & & 3 & & 2 & & & & 5 \\
\hline 213.7 & 343.8 & & & 6 & 1 & 4 & 2 & & & 13 \\
\hline 213.9 & 344.2 & & & 1 & & 1 & & & & 2 \\
\hline 214.5 & 345.1 & & 2 & 2 & 2 & 1 & & & & 7 \\
\hline 215.4 & 346.6 & & & 3 & & & & & & 3 \\
\hline 216.1 & 347.7 & & 1 & 2 & 4 & 2 & & & & 9 \\
\hline 216.9 & 349.0 & & 2 & 4 & 22 & 2 & & & & 30 \\
\hline 217.3 & 349.6 & & 1 & 11 & 11 & 8 & 1 & 4 & & 36 \\
\hline 218.5 & 351.6 & & & 2 & 5 & 3 & & & & 10 \\
\hline 218.7 & 351.9 & & 2 & 4 & 6 & 1 & & & & 13 \\
\hline 219.0 & 352.4 & & 2 & 4 & 4 & 2 & & & & 12 \\
\hline 219.3 & 352.9 & & 4 & 3 & 6 & & & & & 13 \\
\hline 220.7 & 355.1 & & & & 1 & & & & & 1 \\
\hline 222.7 & 358.3 & & & 2 & 8 & 4 & & & & 14 \\
\hline 222.9 & 358.6 & & 2 & 10 & 9 & & & & & 21 \\
\hline
\end{tabular}


Table 2 (Continued)

New fall Chinook salmon redds counted during aerial searches of the Snake River in 2004 (Data collected by Idaho Power Company and the U.S. Fish and Wildlife Service). Counts are presented by river mile (RM), river kilometer (RK), and date. An empty cell indicates no redds were observed on the corresponding location and date. A dash indicates no search was conducted at the corresponding river mile.

\begin{tabular}{|c|c|c|c|c|c|c|c|c|c|c|}
\hline \multirow[b]{2}{*}{$\mathrm{RM}$} & \multirow[b]{2}{*}{ RK } & \multicolumn{8}{|c|}{ New Redds By Flight Date } & \multirow[b]{2}{*}{ Totals } \\
\hline & & 18-Oct & $25-\mathrm{Oct}$ & 1-Nov & 8-Nov & 15-Nov & 22-Nov & 29-Nov & 6-Dec & \\
\hline 224.7 & 361.5 & & 1 & 6 & 3 & 2 & & & & 12 \\
\hline 225.0 & 362.0 & & 1 & & & - & & & - & 1 \\
\hline 231.3 & 372.2 & & & & 2 & - & & & - & 2 \\
\hline 235.0 & 378.1 & & 3 & & 6 & - & & & - & 9 \\
\hline 235.7 & 379.2 & & 5 & & 18 & - & & & - & 23 \\
\hline 236.0 & 379.7 & & & & 2 & - & & & - & 2 \\
\hline 236.1 & 379.9 & & & & 2 & - & & & - & 2 \\
\hline 237.0 & 381.3 & & 2 & & 22 & - & & & - & 24 \\
\hline 238.1 & 383.1 & & & & 1 & - & & & - & 1 \\
\hline 238.6 & 383.9 & & 2 & & 12 & - & & 2 & - & 16 \\
\hline 240.4 & 386.8 & & 2 & & 13 & - & & & - & 15 \\
\hline 240.6 & 387.1 & & 3 & & 29 & - & 4 & & - & 36 \\
\hline 241.0 & 387.8 & & & & 5 & - & 3 & & - & 8 \\
\hline 242.8 & 390.7 & & 3 & & 6 & - & 1 & & - & 10 \\
\hline 243.5 & 391.8 & & & & 10 & - & 2 & & - & 12 \\
\hline 243.8 & 392.3 & & & & 1 & - & 1 & & - & 2 \\
\hline 244.0 & 392.6 & & & & 8 & - & & & - & 8 \\
\hline 244.5 & 393.4 & & & & 25 & - & & & - & 25 \\
\hline 244.7 & 393.7 & & & & & - & 1 & & - & 1 \\
\hline 245.2 & 394.5 & & & & 1 & - & & & - & 1 \\
\hline 245.3 & 394.7 & & & & 7 & - & & & - & 7 \\
\hline 245.7 & 395.3 & & & & 6 & - & & & - & 6 \\
\hline 245.8 & 395.5 & & & & 7 & - & 2 & & - & 9 \\
\hline \multirow[t]{2}{*}{247.5} & 398.2 & & & & & - & 1 & 1 & - & 2 \\
\hline & & 1 & 95 & 303 & 493 & 183 & 82 & 48 & 13 & 1,218 \\
\hline
\end{tabular}


Table 3. Record of fall Chinook salmon redds counted in the Snake River using submersible cameras in 2004 (Data collected by the Idaho Power Company and U.S. Fish and Wildlife Service). Counts are presented by river mile (RM) and river kilometer (RK). In 2004, 49 individual redds were counted in deep water at RM 179.6, and three at RM 158.0. We measured $1,479 \mathrm{~m}^{2}$ of disturbed bottom substrates at RM 179.6, 1,259 $\mathrm{m}^{2}$ at RM 158.0, $981 \mathrm{~m}^{2}$ at RM 193.0, and 1,257 $\mathrm{m}^{2}$ at RM 212.2, equaling 33, 27, 22, and 27 , redds, respectively, using a conversion factor of $45.8 \mathrm{~m}^{2}$-per-redd.

\begin{tabular}{|c|c|c|}
\hline RM & RK & Redds \\
\hline 148.5 & 238.9 & 8 \\
\hline 155.0 & 249.4 & 2 \\
\hline 158.0 & 254.2 & 30 \\
\hline 163.7 & 263.4 & 9 \\
\hline 165.8 & 266.8 & 13 \\
\hline 166.6 & 268.1 & 8 \\
\hline 168.7 & 271.4 & 24 \\
\hline 169.7 & 273.0 & 17 \\
\hline 179.6 & 289.0 & 82 \\
\hline 181.7 & 292.4 & 5 \\
\hline 183.2 & 294.8 & 1 \\
\hline 183.5 & 295.3 & 9 \\
\hline 184.7 & 297.2 & 1 \\
\hline 188.2 & 302.8 & 1 \\
\hline 193.0 & 310.5 & 22 \\
\hline 193.4 & 311.2 & 1 \\
\hline 193.6 & 311.5 & 3 \\
\hline 193.7 & 311.7 & 2 \\
\hline 193.8 & 311.8 & 2 \\
\hline 194.1 & 312.3 & 5 \\
\hline 194.4 & 312.8 & 23 \\
\hline 198.2 & 318.9 & 18 \\
\hline 198.8 & 319.9 & 12 \\
\hline 199.4 & 320.8 & 1 \\
\hline 208.0 & 334.7 & 5 \\
\hline 212.2 & 341.4 & 27 \\
\hline 212.3 & 341.6 & 8 \\
\hline 213.3 & 343.2 & 6 \\
\hline 216.9 & 349.0 & 23 \\
\hline 218.5 & 351.6 & 37 \\
\hline 218.7 & 351.9 & 3 \\
\hline 219.3 & 352.9 & 4 \\
\hline 222.3 & 357.7 & 6 \\
\hline 222.9 & 358.6 & 2 \\
\hline 224.7 & 361.5 & 3 \\
\hline 228.7 & 368.0 & 3 \\
\hline 235.0 & 378.1 & 48 \\
\hline 235.7 & 379.2 & 2 \\
\hline 236.0 & 379.7 & 1 \\
\hline 236.3 & 380.2 & 1 \\
\hline 237.0 & 381.3 & 2 \\
\hline \multirow[t]{2}{*}{242.8} & 390.7 & 11 \\
\hline & & 491 \\
\hline
\end{tabular}


Table 4. List of the 67 sites searched for fall Chinook salmon redds in the Snake River, 2004, by river mile (RM).

\begin{tabular}{cc}
\hline RM & RM \\
\hline 148.5 & 199.4 \\
150.5 & 202.3 \\
151.4 & 203.1 \\
155.0 & 206.6 \\
156.4 & 208.0 \\
158.0 & 208.3 \\
162.4 & 209.9 \\
163.5 & 211.9 \\
164.4 & 212.2 \\
165.7 & 212.3 \\
166.2 & 213.3 \\
166.6 & 215.3 \\
168.8 & 216.9 \\
169.7 & 218.4 \\
171.0 & 218.5 \\
179.5 & 218.7 \\
181.1 & 219.0 \\
181.7 & 219.3 \\
183.2 & 221.0 \\
183.6 & 221.3 \\
184.7 & 222.3 \\
185.6 & 222.8 \\
188.2 & 223.1 \\
192.9 & 224.7 \\
193.4 & 228.1 \\
193.5 & 228.7 \\
193.7 & 229.1 \\
193.8 & 235.1 \\
194.1 & 235.7 \\
194.4 & 236.0 \\
198.2 & 236.3 \\
198.8 & 237.0 \\
& 242.8 \\
\hline
\end{tabular}


Table 5. Flight information, river flow, and visibility ration for aerial surveys of the Snake River in 2004. Flights on 1-Nov, 15-Nov, and 6-Dec, were terminated prior to reaching Hells Canyon Dam (RM 247) due to high winds.

\begin{tabular}{|c|c|c|c|c|c|c|c|c|}
\hline \multirow[b]{2}{*}{ Category } & \multicolumn{8}{|c|}{ Flight Date } \\
\hline & 18-Oct & 25-Oct & 1-Nov & 8-Nov & 15-Nov & 22-Nov & 29-Nov & 6-Dec \\
\hline River Mile Start & 147 & 147 & 147 & 147 & 147 & 147 & 147 & 147 \\
\hline River Mile End & 247 & 247 & 227 & 247 & 224.8 & 247 & 247 & 224.8 \\
\hline Aircraft & H. Soloy & H. Soloy & H. Soloy & H. Soloy & H. Soloy & H. Soloy & H. Soloy & H. Soloy \\
\hline Pilot & J. Pope Jr. & J. Pope Jr. & J. Pope Jr. & J. Pope Jr. & J. Pope Jr. & J. Pope Jr. & J. Pope Jr. & J. Pope Jr. \\
\hline Primary Observer & P. Groves & P. Groves & P. Groves & P. Groves & P. Groves & P. Groves & P. Groves & P. Groves \\
\hline Secondary Observer & S. Bradbury & S. Bradbury & S. Bradbury & S. Bradbury & S. Bradbury & S. Bradbury & S. Bradbury & S. Bradbury \\
\hline Weather & Ptly Cldy & Ptly Cldy & Mstly Cldy & Clear & Mstly Cldy & Cloudy & Ptly Cldy & Mstly Cldy \\
\hline Flow (cfs) at RM 67.5 & 13,800 & 14,500 & 14,700 & 14,400 & 14,400 & 13,700 & 14,600 & 13,400 \\
\hline Flow (cfs) at RM 247.0 & 8,790 & 8,687 & 8,535 & 8,693 & 8,643 & 8,720 & 9,519 & 9,345 \\
\hline Visibility Rating (VR): Asotin to Grande Ronde River & Fair & Fair & Good & Good & Good & Good & Good & Good \\
\hline VR: G.R. River to Salmon River & Fair & Fair & Good & Good & Good & Good & Good & Good \\
\hline VR: Salmon River to H.C. Dam & Fair & Fair & Good & Good & Good & Good & Good & Good \\
\hline
\end{tabular}


Table 6. Number of redd searches conducted in the Snake River and tributaries between Lower Granite and Hells Canyon dams, 1986-2004. Data for underwater searches indicates the number of discrete patches of gravels searched, whereas all other data indicates the number of helicopter flights over portions of the corresponding river

\begin{tabular}{|c|c|c|c|c|c|c|c|c|c|c|c|c|c|c|c|c|c|c|c|}
\hline \multirow[b]{2}{*}{ Location } & \multicolumn{19}{|c|}{ Year } \\
\hline & 1986 & 1987 & 1988 & 1989 & 1990 & 1991 & 1992 & 1993 & 1994 & 1995 & 1996 & 1997 & 1998 & 1999 & 2000 & 2001 & 2002 & 2003 & 2004 \\
\hline Snake (helicopter) & 1 & 2 & 2 & 2 & 3 & 9 & 8 & 8 & 8 & 7 & 7 & 8 & 8 & 9 & 9 & 10 & 7 & 7 & 8 \\
\hline Snake (video) & & & & & & 1 & 3 & 50 & 73 & 42 & 32 & 63 & 48 & 73 & 60 & 67 & 60 & 47 & 67 \\
\hline $\begin{array}{l}\text { Lower Clearwater } \\
\text { (RM 0-41) }\end{array}$ & & & 1 & 2 & 2 & 2 & 2 & 5 & 5 & 3 & 4 & 9 & 5 & 10 & 11 & 8 & 9 & 9 & 10 \\
\hline Potlatch River ${ }^{a}$ & & & & & & & & & & & & & & 1 & 5 & 3 & 3 & 2 & 3 \\
\hline N.F. Clearwater & & & & & & & 2 & 4 & 5 & 3 & 5 & 9 & 5 & 7 & 11 & 4 & 9 & 9 & 10 \\
\hline $\begin{array}{l}\text { Clearwater River } \\
\text { (RM 42-74) }\end{array}$ & & & & & & & & & 5 & 2 & 1 & 7 & 5 & 8 & 11 & 4 & 3 & 9 & 2 \\
\hline S.F. Clearwater & & & & & & & 2 & 4 & 4 & 1 & 3 & 7 & 5 & 8 & 6 & 7 & 3 & 3 & 2 \\
\hline $\begin{array}{l}\text { M.F. Clearwater } \\
\text { (RM 75-98) }\end{array}$ & & & & & & & & & 1 & 2 & 2 & 2 & 5 & 3 & 4 & 5 & 1 & 1 & 0 \\
\hline Selway River & & & & & & & & & 1 & 2 & 2 & 2 & 5 & 3 & 5 & 6 & 1 & 1 & 0 \\
\hline Asotin Creek & & & 1 & 1 & 2 & 1 & & & & & & & & & & & & 2 & 2 \\
\hline Grande Ronde & 1 & 3 & 2 & 1 & 1 & 3 & 6 & 8 & 7 & 3 & 4 & 8 & 6 & 7 & 7 & 9 & 7 & 8 & 8 \\
\hline Salmon River & & & & & & & 2 & 3 & 3 & 1 & 4 & 3 & 3 & 3 & 2 & 1 & 2 & 3 & 3 \\
\hline Imnaha River & & 1 & 2 & 2 & 1 & 9 & 6 & 8 & 8 & 6 & 5 & 7 & 6 & 9 & 9 & 9 & 7 & 8 & 8 \\
\hline
\end{tabular}

${ }^{a}$ Fall Chinook salmon redds were observed in the Potlatch River in 2002-2004 during air and (or) ground searches for coho redds and carcasses. 
Table 7. Numbers of fall Chinook salmon redds counted using submersible cameras in the Snake River, $1991-2004$. Counts are presented by river mile (RM) and river kilometer (RK). A zero indicates the site was searched but no redds were observed. An empty cell indicates the site was not searched in the corresponding year.

\begin{tabular}{|c|c|c|c|c|c|c|c|c|c|c|c|c|c|c|c|}
\hline \multirow[b]{2}{*}{$\mathrm{RM}$} & \multirow[b]{2}{*}{ RK } & \multicolumn{14}{|c|}{ Teal } \\
\hline & & 1991 & 1992 & 1993 & 1994 & 1995 & 1996 & 1997 & 1998 & 1999 & 2000 & 2001 & 2002 & 2003 & 2004 \\
\hline 148.5 & 238.9 & & & 0 & 0 & & 0 & 0 & & 0 & 0 & 1 & 3 & & 8 \\
\hline 155.0 & 249.4 & & & & & & & & & & & & & & 2 \\
\hline 156.4 & 251.6 & & & & & & & & & & & & 18 & 0 & \\
\hline 158.0 & 254.2 & & & 0 & 0 & & 0 & 0 & 0 & 0 & 0 & 0 & 1 & 4 & 30 \\
\hline 162.4 & 261.3 & 5 & 0 & 0 & 0 & & 2 & 0 & 0 & 0 & 0 & 6 & 1 & 0 & \\
\hline 163.7 & 263.4 & & & & 0 & & 0 & 0 & & 0 & 0 & 0 & 0 & 10 & 9 \\
\hline 165.3 & 266.0 & & & 0 & 0 & & & & & & & 4 & 0 & & \\
\hline 165.7 & 266.6 & & & 28 & 0 & & 0 & 0 & 0 & 0 & 0 & 6 & 8 & 48 & 13 \\
\hline 166.2 & 267.4 & & & 11 & 0 & & 0 & 0 & 0 & 0 & 0 & 0 & 1 & 0 & \\
\hline 166.6 & 268.1 & & & 21 & 0 & & 0 & 0 & 6 & 0 & 1 & 0 & 9 & 1 & 8 \\
\hline 168.7 & 271.4 & & & & & & & & & & & & & 28 & 24 \\
\hline 169.7 & 273.0 & & & 0 & 0 & & 0 & 0 & & & & & & 10 & 17 \\
\hline 178.5 & 287.2 & & & & & & & & & & & & & 25 & \\
\hline 179.6 & 289.0 & & & 2 & 8 & 19 & 24 & 5 & 16 & 30 & 48 & 67 & 75 & 94 & 82 \\
\hline 181.8 & 292.5 & & & 0 & 0 & & & 0 & & 1 & & 0 & 0 & & 5 \\
\hline 183.1 & 294.6 & & & 0 & 0 & & & 0 & 0 & 2 & 0 & 0 & 4 & & 1 \\
\hline 183.5 & 295.3 & & & 0 & & & & & & & & & & & 9 \\
\hline 184.7 & 297.2 & & & 0 & 0 & & & 0 & & 0 & 0 & 0 & 0 & 6 & 1 \\
\hline 188.2 & 302.8 & & & & & & & & & 0 & 0 & 2 & 0 & 3 & 1 \\
\hline 193.0 & 310.5 & & & & & & & & & & & & 21 & 16 & 22 \\
\hline 193.4 & 311.2 & & & 0 & & & & & & & & & & & 1 \\
\hline 193.6 & 311.5 & & & 0 & 0 & 0 & 0 & 0 & 0 & 0 & 0 & 4 & 2 & & 3 \\
\hline 193.7 & 311.7 & & 0 & & 0 & 0 & 0 & 0 & 0 & 0 & 0 & 1 & 1 & & 2 \\
\hline 193.8 & 311.8 & & & 1 & 0 & 0 & 0 & 0 & 0 & 0 & 0 & 0 & 1 & & 2 \\
\hline 194.1 & 312.3 & & & & 5 & 0 & 0 & 0 & 0 & & & 0 & 0 & & 5 \\
\hline 194.4 & 312.8 & & & & 0 & 0 & & 0 & & 0 & 0 & 0 & 1 & 5 & 23 \\
\hline 198.2 & 318.9 & & & & 0 & 0 & 2 & 0 & 0 & 6 & 5 & 19 & 11 & 13 & 18 \\
\hline 198.8 & 319.9 & & & & 0 & 0 & 0 & 0 & 0 & 4 & 4 & 2 & 2 & 4 & 12 \\
\hline 199.4 & 320.8 & & & 1 & 0 & 0 & 5 & 0 & 0 & 2 & 1 & 2 & 0 & 0 & 1 \\
\hline 203.1 & 326.8 & & & 0 & 0 & 0 & 0 & 0 & 10 & 0 & 0 & 0 & 0 & 2 & \\
\hline 208.0 & 334.7 & & & 0 & 0 & 0 & 0 & 4 & 0 & 11 & 0 & 0 & 1 & 14 & 5 \\
\hline 208.3 & 335.2 & & & 0 & 0 & 0 & 0 & 0 & 0 & 4 & 0 & 4 & 0 & 0 & \\
\hline 211.9 & 340.9 & & & 0 & & & & & & 1 & & & & 0 & \\
\hline 212.2 & 341.4 & & & 0 & 0 & 2 & 0 & 0 & 17 & 24 & 28 & 37 & 37 & 33 & 27 \\
\hline 212.3 & 341.6 & & & & & 0 & & & 0 & 2 & 3 & 8 & 5 & 2 & 8 \\
\hline 213.3 & 343.2 & & & 0 & 0 & 0 & & 0 & 0 & 0 & 0 & 4 & 8 & 8 & 6 \\
\hline 216.9 & 349.0 & & & & & & & & & 4 & 0 & 1 & & 2 & 23 \\
\hline 218.5 & 351.6 & & & & & & & & & 0 & 1 & 0 & 11 & 1 & 37 \\
\hline 218.7 & 351.9 & & & & & 3 & & 0 & 0 & 1 & & 4 & 2 & 0 & 3 \\
\hline 219.0 & 352.4 & & & & & & & & & 1 & & & 0 & & \\
\hline 219.3 & 352.9 & & & & & & & & & & & & & & 4 \\
\hline 221.0 & 355.6 & & & & & & & & & 0 & 0 & 0 & 1 & 0 & \\
\hline 222.3 & 357.7 & & & 0 & 0 & 0 & & 0 & 0 & 0 & 0 & 0 & 0 & 0 & 6 \\
\hline 222.8 & 358.5 & & & 3 & 0 & 0 & & & & 0 & 0 & & & 0 & 2 \\
\hline 223.1 & 359.0 & & & & & & & & & 3 & 0 & 0 & & & \\
\hline 228.0 & 366.9 & & & & 0 & 0 & 0 & 0 & 0 & 2 & 0 & 0 & 0 & 1 & \\
\hline 224.7 & 361.5 & & & & & & & & & & & & & & 3 \\
\hline 228.7 & 368.0 & & & & 0 & 0 & & 0 & & 0 & & & & & 3 \\
\hline
\end{tabular}


$235.0 \quad 378.1$

$235.7 \quad 379.2$

$236.0 \quad 379.7$

$236.3 \quad 380.2$

$236.9 \quad 381.2$

$237.0 \quad 381.3$

$239.4 \quad 385.2$

$242.9 \quad 390.8$

$245.8 \quad 395.5$

245.8395 .5

Note: Searchers reported $170 \mathrm{~m}^{2}$ patch

tin $1995,238 \mathrm{~m}^{2}$ in 1996 conversion factor of $45.8 \mathrm{~m}^{2}$ per-redd was used to estimate the numbers of redds within these patches. In addition, RM 211.9 and 219 are not deep-water sites. Cameras were used in for ground truthing due to poor observation conditions. 
Table 8. New fall Chinook salmon redds counted in the Clearwater River and North Fork Clearwater River in 2004. Counts are presented by river mile (RM) and date. An empty cell indicates no redds were observed on the corresponding location and date. A dash indicates no search was conducted at the corresponding river mile (Data collected by the Nez Perce Tribe).

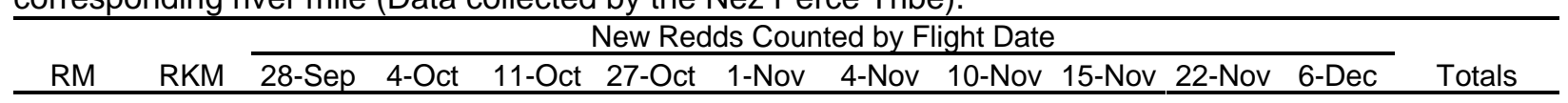

\section{Clearwater River}

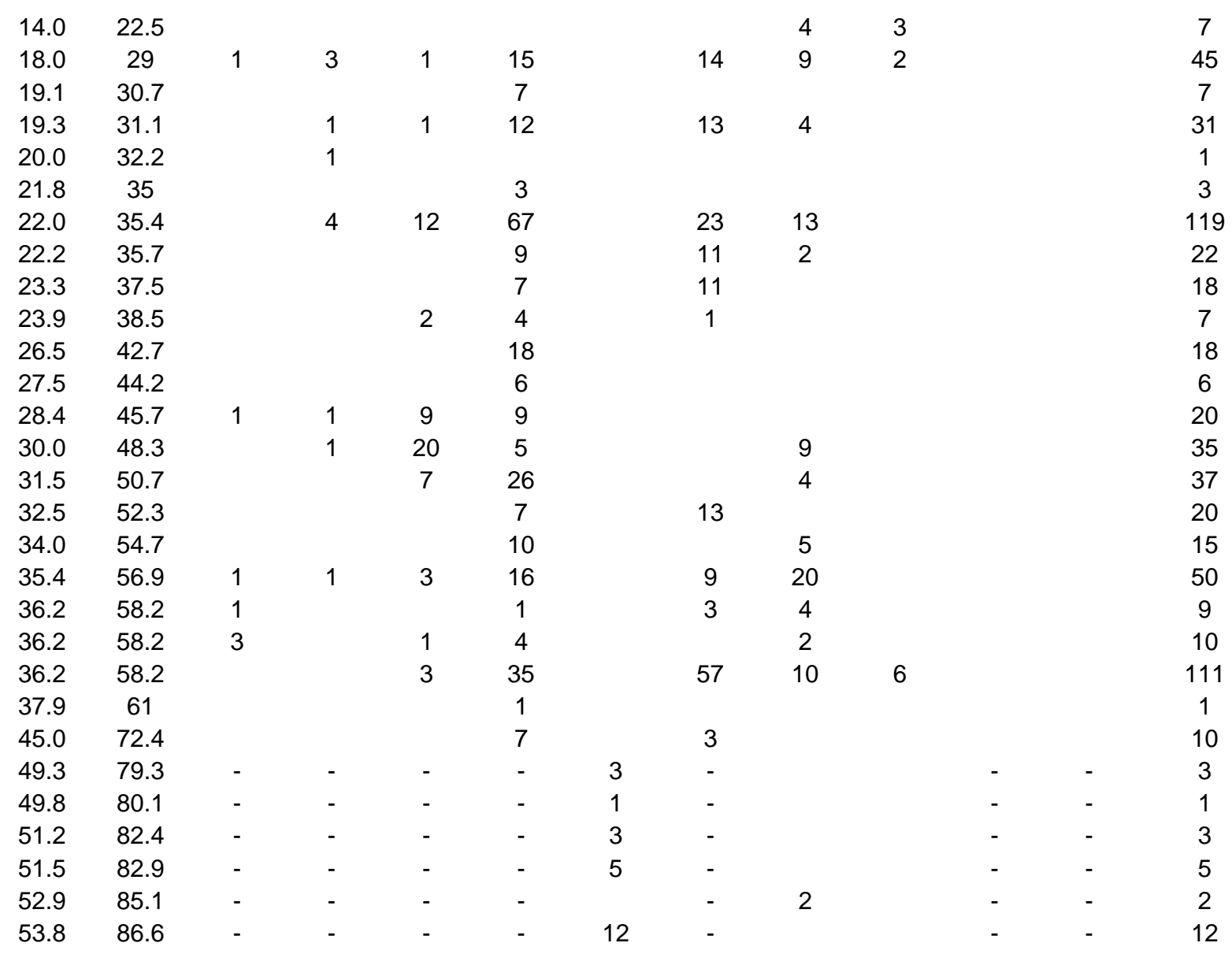

North Fork Clearwater River

$0.2 \quad 0.3$

2

2

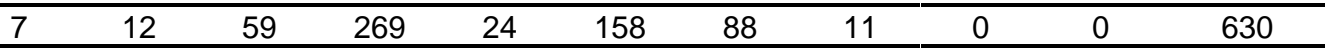


Table 9. Flight information, river flow, and visibility rating for aerial redd surveys of the Clearwater River in 2004 (Data provided by the Nez Perce Tribe).

\begin{tabular}{llccccccccc}
\hline Category & \multicolumn{1}{c}{ Flight date } \\
\cline { 2 - 10 } & 28-Sep & 4-Oct & 11-Oct & 27-Oct & 1-Nov & 4-Nov & 10-Nov & 15-Nov 22-Nov & $6-D e c$ \\
\hline Start (RM) & 4 & 4 & 4 & 4 & 45 & 4 & 4 & 4 & 4 & 4 \\
End (RM) & 45 & 45 & 45 & 45 & 75 & 45 & 54 & 54 & 45 & 75 \\
Flow (cfs) at RM 11.6 & 5,520 & 4,630 & 4,400 & 4,630 & 4,710 & 5,660 & 4,790 & 4,500 & 4,160 & 4,790 \\
Flow (cfs) at RM 37.4 & 1,700 & 1,700 & 1,700 & 1,700 & 1,700 & 1,700 & 1,500 & 1,500 & 1,500 & 1,500 \\
Flow (cfs) at RM 44.6 & 3,680 & 2,820 & 2,580 & 2,860 & 2,860 & 3,880 & 3,050 & 2,910 & 2,420 & 3,040 \\
Visibility rating & Poor & Poor & Poor & Poor & Poor & Poor & Poor & Poor & Poor & Poor \\
\hline
\end{tabular}


Table 10. New fall Chinook salmon redds counted during aerial searches of the Grande Ronde River in 2004 (Data collected by the Nez Perce Tribe). Counts are presented by river mile (RM), river kilometer (RK), and date. An empty cell indicates no redd were observed on the corresponding location. A dash indicates no survey was conducted over the corresponding river mile.

\begin{tabular}{|c|c|c|c|c|c|c|c|c|c|c|}
\hline \multirow[b]{2}{*}{ RM } & \multirow[b]{2}{*}{ RK } & \multicolumn{8}{|c|}{ New Redds Counted by Flight Date } & \multirow[b]{2}{*}{ Totals } \\
\hline & & $12-$ Oct & 19-Oct & 26-Oct & 2-Nov & 10-Nov & 16-Nov & 23-Nov & 30-Nov & \\
\hline 0.1 & 0.2 & & & & & & & 1 & & 1 \\
\hline 1 & 1.6 & & & & & 1 & & & 1 & 2 \\
\hline 2 & 3.2 & & & & 7 & 7 & 9 & 7 & & 30 \\
\hline 2.4 & 3.9 & & & & 2 & & & & & 2 \\
\hline 3.2 & 5.1 & & & & 3 & & & & & 3 \\
\hline 3.3 & 5.3 & & & & 1 & & & & & 1 \\
\hline 3.7 & 6.0 & & & & & 15 & 14 & 2 & & 31 \\
\hline 4.3 & 6.9 & & & 3 & & & 1 & & & 4 \\
\hline 4.4 & 7.1 & & 1 & & 1 & & & & & 2 \\
\hline 4.6 & 7.4 & & & 1 & & & & & & 1 \\
\hline 5.6 & 9.0 & & & & 1 & & & & & 1 \\
\hline 9.1 & 14.6 & & & & 1 & & & & & 1 \\
\hline 10 & 16.1 & & 3 & & & & & & & 3 \\
\hline 10.5 & 16.9 & & & 3 & 1 & 4 & 1 & 4 & & 13 \\
\hline 11.4 & 18.3 & & & & 1 & & & & & 1 \\
\hline 11.7 & 18.8 & & 1 & & & & 1 & & & 2 \\
\hline 12.5 & 20.1 & & & 2 & 1 & & & & & 3 \\
\hline 12.6 & 20.3 & & & 1 & & 1 & & & & 2 \\
\hline 13.4 & 21.6 & & & 2 & 1 & & & & & 3 \\
\hline 13.8 & 22.2 & & 3 & 2 & & 2 & & 1 & & 8 \\
\hline 14.2 & 22.8 & & 1 & & & & 1 & & & 2 \\
\hline 14.3 & 23.0 & & & & & & & & 4 & 4 \\
\hline 16.2 & 26.1 & & & & & 2 & & & & 2 \\
\hline 17.7 & 28.5 & 1 & & & 3 & 2 & & & & 6 \\
\hline 22.1 & 35.6 & & & & & & 1 & & & 1 \\
\hline 22.2 & 35.7 & & & & & & & & 1 & 1 \\
\hline 24.0 & 38.6 & 1 & & & & & & 1 & & 2 \\
\hline 24.4 & 39.3 & & & & 1 & & & & & 1 \\
\hline 26.7 & 43.0 & 1 & & & & & & & & 1 \\
\hline 27.9 & 44.9 & & 1 & & & & & & & 1 \\
\hline 29.1 & 46.8 & & 2 & 2 & & & & & 1 & 5 \\
\hline 31.1 & 50.0 & & & & & & 1 & & & 1 \\
\hline 37.7 & 60.7 & 2 & 2 & & & & & & & 4 \\
\hline 43.2 & 69.5 & & & 1 & & & & & & 1 \\
\hline 44.5 & 71.6 & & & 2 & 1 & & & 1 & & 4 \\
\hline 44.9 & 72.2 & 1 & & & 4 & & & & & 5 \\
\hline 45.7 & 73.5 & 2 & 2 & & & & & & & 4 \\
\hline 48.7 & 78.4 & & & & & & 1 & 1 & & 2 \\
\hline \multirow[t]{2}{*}{49.2} & 79.2 & & & 1 & & & & & & 1 \\
\hline & & 8 & 16 & 20 & 29 & 34 & 30 & 17 & 7 & 162 \\
\hline
\end{tabular}


Table 11. Flight information, river discharge, and visibility rating for aerial redd surveys of the Grande Ronde River in 2004. All surveys were conducted from a Jet Ranger helicopter. Flights conducted by NPT.

\begin{tabular}{lcccccccc}
\hline & \multicolumn{1}{c}{ Flight date } \\
\cline { 2 - 9 } Category & 12-Oct & 19-Oct & 26-Oct & 2-Nov & 10-Nov & 16-Nov & 23-Nov & 30-Nov \\
\hline Start (RM) & 0 & 0 & 0 & 0 & 0 & 0 & 0 & 0 \\
End (RM) & 52.7 & 52.7 & 52.7 & 52.7 & 52.7 & 52.7 & 52.7 & 52.7 \\
Flow (cfs) at RM 45.2 & 738 & 866 & 846 & 806 & 826 & 836 & 806 & 856 \\
Visibility rating & Excel & Good & Fair & Good & Good & Fair & Good & Good \\
\hline
\end{tabular}


Table 12. Fall Chinook aerial spawning ground surveys conducted in the Salmon River, 2004 (Data collected by the Nez Perce Tribe). Counts are presented by river mile (RM), river kilometer (RK), and date. An empty cell indicates no redd were found on the corresponding location and date. A dash (-) indicates the site was not searched on the corresponding date.

\begin{tabular}{cccccc}
\hline & & \multicolumn{3}{c}{ New redds counted by flight date } & Totals \\
\cline { 3 - 6 } RM & RK & 27-Oct & 11-Oct & 24-Nov & \\
\hline & & & & & 6 \\
4.0 & 6.4 & & 2 & & 1 \\
4.8 & 7.7 & 1 & & 1 & 1 \\
24.4 & 39.3 & & 2 & 3 & 8 \\
30.9 & 49.7 & 3 & & & 1 \\
31.0 & 49.9 & 1 & 1 & 1 & 1 \\
35.7 & 57.4 & & & 2 & 1 \\
82.2 & 132.3 & & - & 11 & 2 \\
110.0 & 177.0 & - & 5 & & 21 \\
\hline
\end{tabular}

Table 13. New fall Chinook salmon redds counted during aerial searches of the Imnaha River in 2004 (Data collected by the Nez Perce Tribe). Counts are presented by river mile (RM), river kilometer (RK), and date. An empty cell indicates no survey was conducted over the corresponding river mile, and a dash (-) indicates no redd were observed on the corresponding location and date.

\begin{tabular}{|c|c|c|c|c|c|c|c|c|c|c|}
\hline \multirow[b]{2}{*}{ RM } & \multirow[b]{2}{*}{ RKM } & \multicolumn{8}{|c|}{ New Redds Counted by Flight Date } & \multirow[b]{2}{*}{ Totals } \\
\hline & & 12-Oct & $19-$ Oct & $26-$ Oct & 2-Nov & $10-\mathrm{Nov}$ & $16-\mathrm{Nov}$ & 23-Nov & $30-\mathrm{Nov}$ & \\
\hline 0.4 & 0.6 & & & & & & & 3 & & 3 \\
\hline 0.5 & 0.8 & & & 1 & 2 & 1 & & & & 4 \\
\hline 1.0 & 1.6 & & & & & & 2 & & & 2 \\
\hline 1.2 & 1.9 & & & & & & & 1 & & 1 \\
\hline 1.3 & 2.1 & & & & & & & 1 & & 1 \\
\hline 2.4 & 3.9 & & & 1 & & & & 1 & & 2 \\
\hline 2.7 & 4.3 & & & 1 & & & & 1 & & 2 \\
\hline 2.8 & 4.5 & & & & & 1 & & & & 1 \\
\hline 3.8 & 6.1 & 1 & & & & & & & & 1 \\
\hline 4.4 & 7.1 & & & & & & 1 & & & 1 \\
\hline 6.0 & 9.7 & & & & & 1 & & & & 1 \\
\hline 5.6 & 9.0 & & & 1 & & 1 & & & & 2 \\
\hline 7.0 & 11.3 & & & 1 & & & & & & 1 \\
\hline 7.8 & 12.6 & & & 1 & 2 & 5 & 1 & & & 9 \\
\hline 8.2 & 13.2 & & & & & 1 & & & & 1 \\
\hline 8.9 & 14.3 & & & & & & 1 & & & 1 \\
\hline 9.3 & 15.0 & & & & 1 & & & & & 1 \\
\hline 14.3 & 23.0 & & & & & & & 1 & & 1 \\
\hline Totals & & 1 & 0 & 6 & 5 & 10 & 5 & 8 & 0 & 35 \\
\hline
\end{tabular}


Table 14. Flight information, river flow, and visibility rating for aerial redd surveys of the Imnaha River in 2004.

\begin{tabular}{|c|c|c|c|c|c|c|c|c|}
\hline \multirow[b]{2}{*}{ RM } & \multicolumn{8}{|c|}{ Flight date } \\
\hline & $10 / 12$ & $10 / 19$ & $10 / 26$ & $11 / 2$ & $11 / 10$ & $11 / 16$ & $11 / 23$ & $11 / 30$ \\
\hline$\overline{\text { Start (RM) }}$ & 0 & 0 & 0 & 0 & 0 & 0 & 0 & 0 \\
\hline End (RM) & 4.2 & 19.2 & 19.2 & 18.3 & 19.2 & 34.9 & 19.2 & 19.2 \\
\hline Flow (cfs) at RM 19.3 & 131 & 168 & 157 & 155 & 153 & 148 & 153 & 112 \\
\hline Visibility rating & Excellent & Good & Good & Good & Good & Good & Excellent & Good \\
\hline
\end{tabular}

Table 15. Annual count of adult fall Chinook salmon in the Lower Granite Dam fish ladder (Raw count), the number removed (Adult take), the number estimated to have passed the dam (Adults passed), the number of redds counted upstream of the dam, and number of adult fall Chinook salmon counted per redd counted upstream, 1986-2004. Raw counts are from USACE annual fish passage reports (USACE 1986-2004), and values for adult take are from the Washington Department of Fish and Wildlife (D. Milks, personal communication).

\begin{tabular}{cccccc}
\hline Year & Raw count & Adult take & $\begin{array}{c}\text { Adults } \\
\text { passed }\end{array}$ & $\begin{array}{c}\text { Redds counted } \\
\text { upstream }\end{array}$ & $\begin{array}{c}\text { No. of adults passed } \\
\text { per redd counted } \\
\text { upstream }\end{array}$ \\
\hline 1986 & 784 & 13 & 771 & 7 & 110.1 \\
1987 & 951 & 3 & 948 & 73 & 13.0 \\
1988 & 627 & 2 & 625 & 87 & 7.2 \\
1989 & 706 & 0 & 706 & 69 & 10.2 \\
1990 & 385 & 50 & 335 & 45 & 7.4 \\
1991 & 630 & 40 & 590 & 54 & 10.9 \\
1992 & 855 & 187 & 668 & 82 & 8.1 \\
1993 & 1,170 & 218 & 952 & 219 & 4.3 \\
1994 & 791 & 185 & 606 & 120 & 5.1 \\
1995 & 1,067 & 432 & 635 & 109 & 5.8 \\
1996 & 1,308 & 389 & 919 & 197 & 4.7 \\
1997 & 1,451 & 444 & 1,007 & 189 & 5.3 \\
1998 & 1,909 & 947 & 962 & 303 & 3.2 \\
1999 & 3,381 & 1,519 & 1,862 & 579 & 3.2 \\
2000 & 3,696 & 1,470 & 2,226 & 543 & 4.1 \\
2001 & 8,915 & 2,286 & 6,629 & 1,302 & 5.1 \\
2002 & 12,351 & 2,404 & 9,947 & 1,854 & 5.4 \\
2003 & 11,732 & 489 & 11,243 & 2,241 & 5.0 \\
2004 & 14,960 & 466 & 14,494 & 2,562 & 5.7 \\
\hline
\end{tabular}


Appendices 


\section{Appendix 1}

Redd counts recorded from 1959 to 1978 in the Snake River between Lewiston, Idaho, and the Hells Canyon Dam site.

\begin{tabular}{|c|c|c|c|c|c|c|c|c|c|}
\hline \multirow[b]{2}{*}{ River section } & \multirow[b]{2}{*}{ Citation } & \multicolumn{8}{|c|}{ Year } \\
\hline & & 1959 & 1960 & 1967 & 1969 & - & 1974 & 1975 & 1976 \\
\hline Hells Canyon Dam to Pleasant Valley Dam Site & Irving and Bjornn 1981 & 19 & 2 & 144 & 294 & & & & \\
\hline Pleasant Valley Dam Site to Imnaha River & Irving and Bjornn 1981 & 7 & 2 & 11 & 94 & & & & \\
\hline \multirow[t]{2}{*}{ Imnaha River to Lewiston, ID } & Irving and Bjornn 1981 & 2 & 0 & 33 & 180 & & & & \\
\hline & & 28 & 4 & 188 & 568 & & & & \\
\hline Hells Canyon Dam to Johnson Bar & Witty 1988 & & & & 170 & & 1 & N.D. & 8 \\
\hline Johnson Bar to Pleasant Valley & Witty 1988 & & & & 124 & & 10 & N.D. & 1 \\
\hline Pleasant Valley to Appaloosa & Witty 1988 & & & & 61 & & 3 & N.D. & 0 \\
\hline Appaloosa to Mountain Sheep & Witty 1988 & & & & 33 & & 2 & N.D. & 4 \\
\hline \multirow[t]{2}{*}{ Mountain Sheep to State Line } & Witty 1988 & & & & 0 & & 0 & N.D. & 0 \\
\hline & & & & & 388 & & 16 & 10 & 13 \\
\hline
\end{tabular}

$-\quad 188$

568

16

$10 \quad 13$


Appendix 2

Fall Chinook salmon redds counted in the Snake River during aerial searches, by river mile (RM), river kilometer (RK), and year (1986-2004). An empty cell indicates no redds were observed at the corresponding site and year.

\begin{tabular}{|c|c|c|c|c|c|c|c|c|c|c|c|c|c|c|c|c|c|c|c|c|}
\hline \multirow[b]{2}{*}{$\mathrm{RM}$} & \multirow[b]{2}{*}{ RK } & \multicolumn{19}{|c|}{ Year } \\
\hline & & 1986 & 1987 & 1988 & 1989 & 1990 & 1991 & 1992 & 1993 & 1994 & 1995 & 1996 & 1997 & 1998 & 1999 & 2000 & 2001 & 2002 & 2003 & 2004 \\
\hline 148.0 & 238.1 & & & & & & & & & & & & & & & & & 5 & & \\
\hline 148.3 & 238.6 & & & & & 1 & & & & & & & & & & & & & 1 & \\
\hline 148.5 & 238.9 & & & & & & & & & & & & & & & 5 & 4 & 37 & 22 & 59 \\
\hline 148.8 & 239.4 & & & & 1 & & & & & & & & & & & & & & & \\
\hline 148.9 & 239.6 & & & & & & & & & & & & & & & & & 2 & 4 & \\
\hline 149.1 & 239.9 & & & & 1 & & 2 & & 1 & & & & & 2 & 1 & & 2 & & & \\
\hline 149.2 & 240.1 & & & & & & & & & & & & & & & & 4 & 13 & 15 & \\
\hline 149.4 & 240.4 & & & & & & & & & & & & & & & & & & 1 & 9 \\
\hline 149.6 & 240.7 & & & & & & & & & & & & & & & & & & & 2 \\
\hline 150.0 & 241.4 & & & & & & & & & & & & & & & & & 1 & & \\
\hline 151.5 & 243.8 & & & & & & & & 2 & & & & & & & & 5 & & 4 & 3 \\
\hline 151.9 & 244.4 & & & 1 & & & & & & & 3 & 4 & 8 & & 1 & & & 7 & 31 & 26 \\
\hline 152.1 & 244.7 & & & & & & & & & & & & & & & & 1 & & 6 & \\
\hline 152.3 & 245.1 & & 13 & 15 & 23 & 16 & & 7 & 3 & 5 & & 3 & 12 & 3 & 20 & 21 & 52 & 23 & 19 & 46 \\
\hline 152.4 & 245.2 & & & & & & & & & & & & & & & & & & & 2 \\
\hline 152.8 & 245.9 & & & & & & & & & & & & & & & & & & & 1 \\
\hline 153.2 & 246.5 & & & & & & & & & & & & & & & & & 4 & 1 & 5 \\
\hline 155.0 & 249.4 & & & & & & & & & & & & & & & & & & & 13 \\
\hline 155.2 & 249.7 & & & & & & & & & & & & & & & & & 1 & & \\
\hline 155.9 & 250.8 & & & & & & & & & & & & & & & & & & & 11 \\
\hline 156.4 & 251.6 & & & & & & & & & & & & & & & & & 18 & & 10 \\
\hline 156.6 & 252.0 & & & & & & & & & & & & & & & & & 28 & & \\
\hline 156.8 & 252.3 & & & & & & & & & & & & & & & 1 & 3 & & & \\
\hline 156.9 & 252.5 & & & & 1 & & & & & & & & & & & & & & & \\
\hline 157.2 & 252.9 & & & & & 1 & & & & & & & & & & & & & & \\
\hline 157.4 & 253.3 & 2 & & & & & & & & & & & & & & & & & & \\
\hline 157.6 & 253.6 & & & & & & & & & & & & 1 & 3 & & & & 1 & 2 & 1 \\
\hline 158.0 & 254.2 & & & & & & & & & & & & & & & & 1 & 2 & 13 & 25 \\
\hline 159.3 & 256.3 & & & & & & & & & & & & & & & & 1 & & 1 & \\
\hline
\end{tabular}


Appendix 2 (Continued)

Fall Chinook salmon redds counted in the Snake River during aerial searches, by river mile (RM), river kilometer (RK), and year (1986-2004). An empty cell indicates no redds were observed at the corresponding site and year.

\begin{tabular}{|c|c|c|c|c|c|c|c|c|c|c|c|c|c|c|c|c|c|c|c|c|}
\hline \multirow[b]{2}{*}{$\mathrm{RM}$} & \multirow[b]{2}{*}{ RK } & \multicolumn{19}{|c|}{ Year } \\
\hline & & 1986 & 1987 & 1988 & 1989 & 1990 & 1991 & 1992 & 1993 & 1994 & 1995 & 1996 & 1997 & 1998 & 1999 & 2000 & 2001 & 2002 & 2003 & 2004 \\
\hline 159.5 & 256.6 & & & & & & & & & & & & & & & & 1 & & 2 & \\
\hline 159.7 & 257.0 & & & & & & & 3 & & & & & & & & & & 3 & 1 & 1 \\
\hline 160.5 & 258.2 & & & & & & & & & & & & & & & & 1 & & 2 & \\
\hline 160.8 & 258.7 & & & & & & & & & & & & & & & 1 & & & & \\
\hline 161.0 & 259.0 & & & & & & & 7 & 11 & & 3 & & 7 & 9 & 1 & 7 & 12 & 11 & 20 & 16 \\
\hline 161.8 & 260.3 & & & & & & & & & & & & & & & & 1 & & & \\
\hline 162.4 & 261.3 & & & 2 & 1 & 2 & 20 & 11 & 1 & & & 2 & & & 1 & 4 & 56 & 33 & 2 & 27 \\
\hline 163.0 & 262.3 & & 3 & & & & & & & & & & & & & & & & & \\
\hline 163.3 & 262.7 & & & & & 2 & & & & & & & & & & & & & & \\
\hline 163.7 & 263.4 & & & & & & & & & & & & & & & & & & 24 & 5 \\
\hline 164.4 & 264.5 & & 2 & & & & & & & & & & & & & & & & & \\
\hline 164.7 & 265.0 & & & & 2 & 1 & & & & 1 & & & 1 & & & & 19 & 12 & 25 & 28 \\
\hline 165.2 & 265.8 & & & 5 & & & & & 2 & 3 & & & & & & & 5 & & & \\
\hline 165.3 & 266.0 & & & & & & & & 2 & & & & & 1 & & & & 7 & 19 & 27 \\
\hline 165.5 & 266.3 & & 4 & & & & & & & & & & & & & & & & & \\
\hline 165.7 & 266.6 & & & & & & & & 28 & & & & & & & & 38 & 52 & 117 & 60 \\
\hline 165.9 & 266.9 & & 2 & 14 & & & 1 & 3 & 9 & & & 3 & & 2 & 5 & & & & & \\
\hline 166.2 & 267.4 & & & & & & & & 17 & & & & & & & & & 1 & & 1 \\
\hline 166.4 & 267.7 & & & & & & & & & & & & & & & & & 9 & & \\
\hline 166.6 & 268.1 & & & & & & 6 & & 21 & & & & & 6 & & 1 & & & 1 & \\
\hline 167.9 & 270.2 & & & & & & & & & & & & & & & & & 1 & & \\
\hline 168.1 & 270.5 & & & & & & & & & & & & & & & & & 1 & & \\
\hline 168.6 & 271.3 & & & & & & & & & & & & & & & & & 1 & 8 & \\
\hline 168.7 & 271.4 & & & & & & & & 5 & 6 & 3 & & & & & & 7 & 47 & 90 & 48 \\
\hline 169.7 & 273.0 & & & & 1 & & & & & & 1 & 1 & & & & & & 11 & 31 & 6 \\
\hline 169.9 & 273.4 & & & & & & & & & & & & & & & & & & 1 & \\
\hline 171.9 & 276.6 & & & & & & & & & & & & & & & & & & & 1 \\
\hline 172.5 & 277.6 & & 1 & & & & & & & & & 3 & & 4 & 1 & 1 & 18 & 11 & 1 & 11 \\
\hline 173.9 & 279.8 & & 1 & & & & & & & & & & & & & 1 & 1 & & 2 & 9 \\
\hline 175.2 & 281.9 & & & & & & & & & & & & & & & & 2 & & & \\
\hline
\end{tabular}


Appendix 2 (Continued)

Fall Chinook salmon redds counted in the Snake River during aerial searches, by river mile (RM), river kilometer (RK), and year (1986-2004). An empty cell indicates no redds were observed at the corresponding site and year.

\begin{tabular}{|c|c|c|c|c|c|c|c|c|c|c|c|c|c|c|c|c|c|c|c|c|}
\hline \multirow[b]{2}{*}{$\mathrm{RM}$} & \multirow[b]{2}{*}{ RK } & \multicolumn{19}{|c|}{ Year } \\
\hline & & 1986 & 1987 & 1988 & 1989 & 1990 & 1991 & 1992 & 1993 & 1994 & 1995 & 1996 & 1997 & 1998 & 1999 & 2000 & 2001 & 2002 & 2003 & 2004 \\
\hline 175.7 & 282.7 & & & & & & & & & & & & & & & & & 1 & 1 & \\
\hline 175.9 & 283.0 & & & & & & & & & & & & & & & & & & & 4 \\
\hline 176.5 & 284.0 & & & & & & & & & & 1 & & & & & 2 & 1 & & 2 & \\
\hline 176.7 & 284.3 & & & & & & & & & & & & & & & & & & & 7 \\
\hline 177.9 & 286.2 & & & & & & & & & & & & & & & & & & & 1 \\
\hline 178.3 & 286.9 & & & & & & & & & 1 & & & & & & & 3 & & & 10 \\
\hline 178.5 & 287.2 & & & & & & & & & & & & & & & 2 & & & 38 & 1 \\
\hline 178.9 & 287.9 & & & & 1 & & & & & 1 & & & & 2 & 7 & 13 & & 18 & 10 & 11 \\
\hline 179.5 & 288.8 & & & & & & & & & & & & & & & & & & & 6 \\
\hline 179.6 & 289.0 & & & & & & & & 6 & 13 & 21 & 32 & 5 & 16 & 40 & 56 & 72 & 92 & 110 & 17 \\
\hline 181.7 & 292.4 & & & & & & & & & & & & & & 1 & 1 & 1 & 3 & 9 & 10 \\
\hline 182.3 & 293.3 & & & & & & & & & & & & & & & & & & 8 & \\
\hline 183.1 & 294.6 & & & & & & & & & & & & & & 2 & & & 4 & & 1 \\
\hline 183.4 & 295.1 & & & & & & & & & & & & & & & & & & 1 & 8 \\
\hline 183.5 & 295.3 & & & & & & & & & & & & & & & & & & 11 & \\
\hline 184.7 & 297.2 & & & & & & & & & & & & & & & & & & 6 & \\
\hline 186.7 & 300.4 & & & & & & & & & & & & & & & & & 3 & & \\
\hline 187.5 & 301.7 & & & & & & & & & & & & & & & & 1 & 1 & & \\
\hline 187.7 & 302.0 & & & & & & & & & & & & & & & & 1 & & 7 & 3 \\
\hline 188.2 & 302.8 & & & & & & & & & & & & & & 1 & 2 & 7 & 1 & 3 & 1 \\
\hline 190.0 & 305.7 & & & & & & & & & & 1 & & & & & & & & 1 & 1 \\
\hline 190.1 & 305.9 & & & & & & & & & 1 & & & & & & & 2 & 5 & 3 & \\
\hline 190.2 & 306.0 & & & & & & & & & & & & & & & & & & & 2 \\
\hline 190.7 & 306.8 & & & & & & & & & & & & & & & & & & 3 & \\
\hline 190.8 & 307.0 & & 1 & 5 & & 2 & 5 & 1 & & & & 1 & & 4 & 2 & 5 & 18 & 28 & 6 & 17 \\
\hline 191.0 & 307.3 & & & & & & & & & & & & & & & & & & 2 & 2 \\
\hline 191.1 & 307.5 & & & & & & & & & & & & & & & & & & & 1 \\
\hline 191.7 & 308.4 & 2 & 2 & 4 & & & & & & & & & & & 1 & & 3 & 10 & 11 & 6 \\
\hline 193.0 & 310.5 & & & & & & & & & & & & & & & & & 28 & 18 & \\
\hline 193.2 & 310.9 & & & & & & & & & & & & & & & & & & 1 & \\
\hline
\end{tabular}


Appendix 2 (Continued)

Fall Chinook salmon redds counted in the Snake River during aerial searches, by river mile (RM), river kilometer (RK), and year (1986-2004). An empty cell indicates no redds were observed at the corresponding site and year.

\begin{tabular}{|c|c|c|c|c|c|c|c|c|c|c|c|c|c|c|c|c|c|c|c|c|}
\hline \multirow[b]{2}{*}{$\mathrm{RM}$} & \multirow[b]{2}{*}{$\mathrm{RK}$} & \multicolumn{19}{|c|}{ Year } \\
\hline & & 1986 & 1987 & 1988 & 1989 & 1990 & 1991 & 1992 & 1993 & 1994 & 1995 & 1996 & 1997 & 1998 & 1999 & 2000 & 2001 & 2002 & 2003 & 2004 \\
\hline 193.4 & 311.2 & & & & 5 & 2 & & & & 2 & & 2 & 1 & 4 & & & & 5 & 3 & \\
\hline 193.6 & 311.5 & & & & & & & & & & & & & & & & 6 & 1 & 9 & 3 \\
\hline 193.7 & 311.7 & & 4 & & & & & 6 & 1 & 2 & 1 & & 2 & 3 & & 5 & 8 & 8 & & 9 \\
\hline 193.8 & 311.8 & & & & & & & & 1 & 1 & 1 & & & & 1 & 2 & 1 & 1 & & 1 \\
\hline 194.0 & 312.1 & & 2 & & & 3 & & & 1 & 2 & 4 & 2 & 6 & 14 & 11 & 11 & 22 & 26 & 20 & 21 \\
\hline 194.1 & 312.3 & & & 2 & & 2 & & & & 5 & & & & & & & 1 & & 1 & 1 \\
\hline 194.4 & 312.8 & & & & & & & & & & & & & & & & & 1 & 5 & \\
\hline 196.0 & 315.4 & & & 3 & & & & & & 2 & & & & 1 & 6 & 10 & 20 & 23 & 34 & 29 \\
\hline 196.2 & 315.7 & & 1 & & & & & & & & & & & & & & & & 25 & \\
\hline 198.2 & 318.9 & & & & & & & & & & & 2 & & 1 & 17 & 14 & 36 & 33 & 13 & 17 \\
\hline 198.8 & 319.9 & & 5 & & 3 & 2 & 7 & 3 & & 6 & 1 & 6 & & 4 & 15 & 17 & 21 & 25 & 39 & 31 \\
\hline 199.4 & 320.8 & & & & & & & & 1 & & & 5 & & & 2 & 1 & 2 & & & \\
\hline 201.0 & 323.4 & & & & & & & & & & & & & & & & & & & 4 \\
\hline 201.1 & 323.6 & & & & & & & & & & & & & & 1 & 1 & 2 & 3 & 6 & \\
\hline 203.1 & 326.8 & & & & & & & & & & & & & 10 & & & & 1 & 10 & \\
\hline 204.1 & 328.4 & & 1 & & & & & & & & & & & & & & & & & \\
\hline 205.3 & 330.3 & & & & & & 3 & & & & 1 & & & 3 & 6 & 2 & & 11 & 17 & 12 \\
\hline 205.4 & 330.5 & & 1 & & & & & & & & & 2 & & & & 4 & & 2 & 4 & 5 \\
\hline 205.7 & 331.0 & & & & & & & & & & & & & & & & & & 2 & \\
\hline 206.4 & 332.1 & & 1 & 4 & & & 1 & 2 & 1 & & 2 & & & 2 & 4 & & 2 & 2 & 10 & 15 \\
\hline 206.6 & 332.4 & & & & & & & & & & & & & & 2 & 1 & & 1 & 5 & 2 \\
\hline 207.5 & 333.9 & & & & & & & & & & & & & & & & & & & 1 \\
\hline 207.7 & 334.2 & & & & & & & & & & & & & & 2 & & & 3 & 3 & \\
\hline 207.8 & 334.4 & & & 1 & & & & & & & & 3 & 2 & & 5 & & & 1 & 3 & 4 \\
\hline 207.9 & 334.5 & & & 2 & & & & & & & & & & & & & & & & \\
\hline 208.0 & 334.7 & & & & 1 & & & & & & 2 & 9 & 5 & 13 & 36 & 17 & 26 & 37 & 62 & 46 \\
\hline 208.1 & 334.8 & & & & & & & & & & & & & & & & & 1 & & \\
\hline 208.3 & 335.2 & & & & & & & & & & & & & & 4 & & & & & \\
\hline 209.1 & 336.4 & & & & & & & & & & & & & & 1 & & & 1 & & \\
\hline 209.7 & 337.4 & & 1 & & & & & & & & & & & & 1 & & & 2 & & \\
\hline
\end{tabular}


Appendix 2 (Continued)

Fall Chinook salmon redds counted in the Snake River during aerial searches, by river mile (RM), river kilometer (RK), and year (1986-2004). An empty cell indicates no redds were observed at the corresponding site and year.

\begin{tabular}{|c|c|c|c|c|c|c|c|c|c|c|c|c|c|c|c|c|c|c|c|c|}
\hline \multirow[b]{2}{*}{$\mathrm{RM}$} & \multirow[b]{2}{*}{ RK } & \multicolumn{19}{|c|}{ Year } \\
\hline & & 1986 & 1987 & 1988 & 1989 & 1990 & 1991 & 1992 & 1993 & 1994 & 1995 & 1996 & 1997 & 1998 & 1999 & 2000 & 2001 & 2002 & 2003 & 2004 \\
\hline 210.8 & 339.2 & & & & & & & & & & & & & & & & & & 1 & \\
\hline 211.9 & 340.9 & & & & & & & & 2 & & & & & 11 & 10 & 6 & 14 & 25 & 30 & 28 \\
\hline 212.2 & 341.4 & & & & & & & & & & 2 & & & 17 & 24 & 28 & 37 & 37 & 34 & \\
\hline 212.3 & 341.6 & & & & & & & & & & & & & & 2 & 3 & 9 & 9 & 4 & 4 \\
\hline 213.3 & 343.2 & & & & 2 & & & & & & & 1 & & & & 1 & 6 & 9 & 12 & \\
\hline 213.5 & 343.5 & & & & & & & & & & & 1 & & & 2 & 1 & 2 & 4 & 6 & 5 \\
\hline 213.7 & 343.8 & & & & & 1 & & 2 & & & & & & 4 & 1 & 1 & 6 & 7 & 1 & 13 \\
\hline 213.9 & 344.2 & & & & & & & & & & & & & & & & & & & 2 \\
\hline 214.5 & 345.1 & & & & & & & & 1 & & & & & & & & 1 & 4 & 3 & 7 \\
\hline 214.7 & 345.5 & & 2 & & & & & & & & & & & & & & & & & \\
\hline 215.4 & 346.6 & & & & & & & & & & & & & 1 & 2 & & & & 3 & 3 \\
\hline 216.1 & 347.7 & & & & & & & & & 1 & & & & 3 & 1 & 2 & 6 & 4 & 6 & 9 \\
\hline 216.9 & 349.0 & & & & & & & & & & & & & & 4 & 5 & 7 & 21 & 23 & 30 \\
\hline 217.3 & 349.6 & & & & & & & 1 & 3 & & 1 & & & 4 & 24 & 6 & 13 & 22 & 42 & 36 \\
\hline 217.8 & 350.4 & & & & 1 & & & & & & & & & & & & & & 2 & \\
\hline 218.2 & 351.1 & & 1 & & & & & & & & & & & & & & & & & \\
\hline 218.5 & 351.6 & & & & & & & & & & & & & & 3 & 3 & & 16 & 11 & 10 \\
\hline 218.6 & 351.7 & & & & & & & & & & & & & & 1 & & & & & \\
\hline 218.7 & 351.9 & & & & & & & & & & 4 & 7 & & 4 & 12 & 5 & 15 & 17 & 20 & 13 \\
\hline 219.0 & 352.4 & & & & & & & & & & 3 & 2 & & 4 & 5 & 6 & 3 & 11 & 7 & 12 \\
\hline 219.3 & 352.9 & & & 2 & & & & 1 & & 3 & & 2 & 3 & 6 & 8 & 5 & 7 & 13 & 12 & 13 \\
\hline 220.7 & 355.1 & & & & & & & & & & & & & & & & & & 4 & 1 \\
\hline 221.0 & 355.6 & & & & & & & & & & & & & & & & & 1 & & \\
\hline 221.5 & 356.4 & & & & & & & & & & & & & & & & 1 & & & \\
\hline 222.3 & 357.7 & & & & & & & & & & & & & & & & & 2 & & \\
\hline 222.7 & 358.3 & & & & 1 & & & & & & & & & & 6 & & 1 & & 8 & 14 \\
\hline 222.8 & 358.5 & 2 & 3 & & & & & & & & & & & & & 5 & & & & \\
\hline 222.9 & 358.6 & & & & 3 & & & & 3 & 1 & & & & & 9 & & 4 & 20 & 19 & 21 \\
\hline 223.2 & 359.1 & & & & & & & & & 3 & 3 & & & & 3 & & & 1 & & \\
\hline 223.7 & 359.9 & & & 1 & & & & & & & & & & & & & & & & \\
\hline
\end{tabular}


Appendix 2 (Continued)

Fall Chinook salmon redds counted in the Snake River during aerial searches, by river mile (RM), river kilometer (RK), and year (1986-2004). An empty cell indicates no redds were observed at the corresponding site and year.

\begin{tabular}{|c|c|c|c|c|c|c|c|c|c|c|c|c|c|c|c|c|c|c|c|c|}
\hline \multirow[b]{2}{*}{$\mathrm{RM}$} & \multirow[b]{2}{*}{$\mathrm{RK}$} & \multicolumn{19}{|c|}{ Year } \\
\hline & & 1986 & 1987 & 1988 & 1989 & 1990 & 1991 & 1992 & 1993 & 1994 & 1995 & 1996 & 1997 & 1998 & 1999 & 2000 & 2001 & 2002 & 2003 & 2004 \\
\hline 224.7 & 361.5 & & & & & & & & & & & & & 3 & & & & & 12 & 12 \\
\hline 225.0 & 362.0 & & & & & & & & & & & & & & 2 & & & 2 & & 1 \\
\hline 225.1 & 362.2 & & & & & & & & & & & & & & 3 & & & 1 & 3 & \\
\hline 226.7 & 364.8 & & & & & & & & & & & & & & 2 & & & & & \\
\hline 228.0 & 366.9 & & & & & & & & & & & & & & 2 & & & & 1 & \\
\hline 231.3 & 372.2 & & & & & & & & & & & & & & & & & 1 & 1 & 2 \\
\hline 235.0 & 378.1 & & & & & & & & & & & & & & & & & & & 9 \\
\hline 235.1 & 378.3 & & & & & & & & & & 1 & & & 1 & 2 & 3 & 8 & 21 & 66 & \\
\hline 235.7 & 379.2 & & 4 & & 3 & & & & & 5 & 2 & 7 & 1 & 4 & 11 & 16 & 16 & 22 & 16 & 23 \\
\hline 236.0 & 379.7 & 1 & 1 & 2 & 1 & & & & & & & & & & 2 & 1 & & & 2 & 2 \\
\hline 236.1 & 379.9 & & & & & & & & & & & & & & & 1 & 1 & 1 & & 2 \\
\hline 236.7 & 380.9 & & 1 & & & & & & & & & & & & & & & & & \\
\hline 237.0 & 381.3 & & & & & & & & 5 & 3 & 1 & & 2 & 8 & 6 & 13 & 14 & 26 & 20 & 24 \\
\hline 238.1 & 383.1 & & & & & & & & & & & & & & & & & & & 1 \\
\hline 238.3 & 383.4 & & & & & & & & & & & & & & 1 & 1 & & 2 & 1 & \\
\hline 238.6 & 383.9 & & 2 & & 2 & & & & & & 1 & & & & 4 & 4 & 8 & 13 & 23 & 16 \\
\hline 239.4 & 385.2 & & & & & & & & & & & & & & & & & & 6 & \\
\hline 240.4 & 386.8 & & & & & & & & & & & & & & & & & & & 15 \\
\hline 240.5 & 387.0 & & 6 & & & & & & & & & 1 & & 2 & 8 & 1 & 3 & 11 & 11 & 36 \\
\hline 240.7 & 387.3 & & & & 3 & & 6 & & & & & 1 & 1 & 4 & 7 & 11 & 13 & 21 & 24 & \\
\hline 241.0 & 387.8 & & & & & & & & & & & & 1 & & & & 4 & 5 & 8 & 8 \\
\hline 242.8 & 390.7 & & & & & & & & & & & & & 1 & & 4 & 4 & 9 & 13 & 10 \\
\hline 243.0 & 391.0 & & & & & & & & & & & & & & 2 & & & & & \\
\hline 243.3 & 391.5 & & 1 & & 1 & & & & & & & & & & & 4 & & & & \\
\hline 243.5 & 391.8 & & & & & & & & & & & 2 & & 1 & & & 5 & & 13 & 12 \\
\hline 243.8 & 392.3 & & & & & & & & & & & & & & & & & & 2 & 2 \\
\hline 244.0 & 392.6 & & & & & & & & & & & & & & 2 & & & 12 & & 8 \\
\hline 244.3 & 393.1 & & & & & & & & & & & & & & & & & 2 & & \\
\hline 244.5 & 393.4 & & & & & & & & & & & & & & & & & & & 25 \\
\hline 244.6 & 393.6 & & & & 1 & 2 & & & & & & & & & 1 & 2 & 9 & 13 & 31 & \\
\hline
\end{tabular}


Appendix 2 (Continued)

Fall Chinook salmon redds counted in the Snake River during aerial searches, by river mile (RM), river kilometer (RK), and year (1986-2004). An empty cell indicates no redds were observed at the corresponding site and year.

\begin{tabular}{|c|c|c|c|c|c|c|c|c|c|c|c|c|c|c|c|c|c|c|c|c|}
\hline \multirow[b]{2}{*}{$\mathrm{RM}$} & \multirow[b]{2}{*}{ RK } & \multicolumn{19}{|c|}{ Year } \\
\hline & & 1986 & 1987 & 1988 & 1989 & 1990 & 1991 & 1992 & 1993 & 1994 & 1995 & 1996 & 1997 & 1998 & 1999 & 2000 & 2001 & 2002 & 2003 & 2004 \\
\hline 244.7 & 393.7 & & & & & & & & & & & & & & & & & & 2 & 1 \\
\hline 245.2 & 394.5 & & & & & & & & & & & & & & & & & & & 1 \\
\hline 245.3 & 394.7 & & & & & & & & & & & & & & 1 & & 1 & & 9 & 7 \\
\hline 245.7 & 395.3 & & & & & & & & & & 2 & & & & & & & & 2 & 6 \\
\hline 245.8 & 395.5 & & & & & & & & & & & & & & 2 & & 1 & 4 & 6 & 9 \\
\hline 246.5 & 396.6 & & & 1 & & & & & & & & & & & & & & & & \\
\hline \multirow[t]{2}{*}{247.5} & 398.2 & & & & & & & & & & & & & & & & & & 4 & 2 \\
\hline & & 7 & 66 & 64 & 58 & 37 & 51 & 47 & 127 & 67 & 65 & 104 & 58 & 185 & 373 & 346 & 709 & 1,113 & 1,512 & 1,218 \\
\hline
\end{tabular}


Appendix 3

Fall Chinook salmon redds counted in the Snake River during aerial and underwater searches, by river mile (RM), river kilometer (RK), and year (19862004). An empty cell indicates no redds were observed at the corresponding site and year.

\begin{tabular}{|c|c|c|c|c|c|c|c|c|c|c|c|c|c|c|c|c|c|c|c|c|}
\hline \multirow[b]{2}{*}{$\mathrm{RM}$} & \multirow[b]{2}{*}{ RK } & \multicolumn{19}{|c|}{ Year } \\
\hline & & 1986 & 1987 & 1988 & 1989 & 1990 & 1991 & 1992 & 1993 & 1994 & 1995 & 1996 & 1997 & 1998 & 1999 & 2000 & 2001 & 2002 & 2003 & 2004 \\
\hline 148.0 & 238.1 & & & & & & & & & & & & & & & & & 5 & & \\
\hline 148.3 & 238.6 & & & & & 1 & & & & & & & & & & & & & 1 & \\
\hline 148.5 & 238.9 & & & & & & & & & & & & & & & 5 & 4 & 37 & 22 & 67 \\
\hline 148.8 & 239.4 & & & & 1 & & & & & & & & & & & & & & & \\
\hline 148.9 & 239.6 & & & & & & & & & & & & & & & & & 2 & 4 & \\
\hline 149.1 & 239.9 & & & & 1 & & 2 & & 1 & & & & & 2 & 1 & & 2 & & & \\
\hline 149.2 & 240.1 & & & & & & & & & & & & & & & & 4 & 13 & 15 & \\
\hline 149.4 & 240.4 & & & & & & & & & & & & & & & & & & 1 & 9 \\
\hline 149.6 & 240.7 & & & & & & & & & & & & & & & & & & & 2 \\
\hline 150.0 & 241.4 & & & & & & & & & & & & & & & & & 1 & & \\
\hline 151.5 & 243.8 & & & & & & & & 2 & & & & & & & & 5 & & 4 & 3 \\
\hline 151.9 & 244.4 & & & 1 & & & & & & & 3 & 4 & 8 & & 1 & & & 7 & 31 & 26 \\
\hline 152.1 & 244.7 & & & & & & & & & & & & & & & & 1 & & 6 & \\
\hline 152.3 & 245.1 & & 13 & 15 & 23 & 16 & & 7 & 3 & 5 & & 3 & 12 & 3 & 20 & 21 & 52 & 23 & 19 & 46 \\
\hline 152.4 & 245.2 & & & & & & & & & & & & & & & & & & & 2 \\
\hline 152.8 & 245.9 & & & & & & & & & & & & & & & & & & & 1 \\
\hline 153.2 & 246.5 & & & & & & & & & & & & & & & & & 4 & 1 & 5 \\
\hline 155.0 & 249.4 & & & & & & & & & & & & & & & & & & & 15 \\
\hline 155.2 & 249.7 & & & & & & & & & & & & & & & & & 1 & & \\
\hline 155.9 & 250.8 & & & & & & & & & & & & & & & & & & & 11 \\
\hline 156.4 & 251.6 & & & & & & & & & & & & & & & & & 18 & & 10 \\
\hline 156.6 & 252.0 & & & & & & & & & & & & & & & & & 28 & & \\
\hline 156.8 & 252.3 & & & & & & & & & & & & & & & 1 & 3 & & & \\
\hline 156.9 & 252.5 & & & & 1 & & & & & & & & & & & & & & & \\
\hline 157.2 & 252.9 & & & & & 1 & & & & & & & & & & & & & & \\
\hline 157.4 & 253.3 & 2 & & & & & & & & & & & & & & & & & & \\
\hline 157.6 & 253.6 & & & & & & & & & & & & 1 & 3 & & & & 1 & 2 & 1 \\
\hline 158.0 & 254.2 & & & & & & & & & & & & & & & & 1 & 2 & 13 & 55 \\
\hline 159.3 & 256.3 & & & & & & & & & & & & & & & & 1 & & 1 & \\
\hline
\end{tabular}


Appendix 3 (Continued)

Fall Chinook salmon redds counted in the Snake River during aerial and underwater searches, by river mile (RM), river kilometer (RK), and year (19862004). An empty cell indicates no redds were observed at the corresponding site and year.

\begin{tabular}{|c|c|c|c|c|c|c|c|c|c|c|c|c|c|c|c|c|c|c|c|c|}
\hline \multirow[b]{2}{*}{$\mathrm{RM}$} & \multirow[b]{2}{*}{ RK } & \multicolumn{19}{|c|}{ Year } \\
\hline & & 1986 & 1987 & 1988 & 1989 & 1990 & 1991 & 1992 & 1993 & 1994 & 1995 & 1996 & 1997 & 1998 & 1999 & 2000 & 2001 & 2002 & 2003 & 2004 \\
\hline 159.5 & 256.6 & & & & & & & & & & & & & & & & 1 & & 2 & \\
\hline 159.7 & 257.0 & & & & & & & 3 & & & & & & & & & & 3 & 1 & 1 \\
\hline 160.5 & 258.2 & & & & & & & & & & & & & & & & 1 & & 2 & \\
\hline 160.8 & 258.7 & & & & & & & & & & & & & & & 1 & & & & \\
\hline 161.0 & 259.0 & & & & & & & 7 & 11 & & 3 & & 7 & 9 & 1 & 7 & 12 & 11 & 20 & 16 \\
\hline 161.8 & 260.3 & & & & & & & & & & & & & & & & 1 & & & \\
\hline 162.4 & 261.3 & & & 2 & 1 & 2 & 20 & 11 & 1 & & & 2 & & & 1 & 4 & 56 & 33 & 2 & 27 \\
\hline 163.0 & 262.3 & & 3 & & & & & & & & & & & & & & & & & \\
\hline 163.3 & 262.7 & & & & & 2 & & & & & & & & & & & & & & \\
\hline 163.7 & 263.4 & & & & & & & & & & & & & & & & & & 24 & 14 \\
\hline 164.4 & 264.5 & & 2 & & & & & & & & & & & & & & & & & \\
\hline 164.7 & 265.0 & & & & 2 & 1 & & & & 1 & & & 1 & & & & 19 & 12 & 25 & 28 \\
\hline 165.2 & 265.8 & & & 5 & & & & & 2 & 3 & & & & & & & 5 & & & \\
\hline 165.3 & 266.0 & & & & & & & & 2 & & & & & 1 & & & & 7 & 19 & 27 \\
\hline 165.5 & 266.3 & & 4 & & & & & & & & & & & & & & & & & \\
\hline 165.7 & 266.6 & & & & & & & & 28 & & & & & & & & 38 & 52 & 117 & 73 \\
\hline 165.9 & 266.9 & & 2 & 14 & & & 1 & 3 & 9 & & & 3 & & 2 & 5 & & & & & \\
\hline 166.2 & 267.4 & & & & & & & & 17 & & & & & & & & & 1 & & 1 \\
\hline 166.4 & 267.7 & & & & & & & & & & & & & & & & & 9 & & \\
\hline 166.6 & 268.1 & & & & & & 6 & & 21 & & & & & 6 & & 1 & & & 1 & 8 \\
\hline 167.9 & 270.2 & & & & & & & & & & & & & & & & & 1 & & \\
\hline 168.1 & 270.5 & & & & & & & & & & & & & & & & & 1 & & \\
\hline 168.6 & 271.3 & & & & & & & & & & & & & & & & & 1 & 8 & \\
\hline 168.7 & 271.4 & & & & & & & & 5 & 6 & 3 & & & & & & 7 & 47 & 90 & 72 \\
\hline 169.7 & 273.0 & & & & 1 & & & & & & 1 & 1 & & & & & & 11 & 31 & 23 \\
\hline 169.9 & 273.4 & & & & & & & & & & & & & & & & & & 1 & \\
\hline 171.9 & 276.6 & & & & & & & & & & & & & & & & & & & 1 \\
\hline 172.5 & 277.6 & & 1 & & & & & & & & & 3 & & 4 & 1 & 1 & 18 & 11 & 1 & 11 \\
\hline
\end{tabular}


Appendix 3 (Continued)

Fall Chinook salmon redds counted in the Snake River during aerial and underwater searches, by river mile (RM), river kilometer (RK), and year (19862004). An empty cell indicates no redds were observed at the corresponding site and year.

\begin{tabular}{|c|c|c|c|c|c|c|c|c|c|c|c|c|c|c|c|c|c|c|c|c|}
\hline \multirow[b]{2}{*}{$\mathrm{RM}$} & \multirow[b]{2}{*}{ RK } & \multicolumn{19}{|c|}{ Year } \\
\hline & & 1986 & 1987 & 1988 & 1989 & 1990 & 1991 & 1992 & 1993 & 1994 & 1995 & 1996 & 1997 & 1998 & 1999 & 2000 & 2001 & 2002 & 2003 & 2004 \\
\hline 173.9 & 279.8 & & 1 & & & & & & & & & & & & & 1 & 1 & & 2 & 9 \\
\hline 175.2 & 281.9 & & & & & & & & & & & & & & & & 2 & & & \\
\hline 175.7 & 282.7 & & & & & & & & & & & & & & & & & 1 & 1 & \\
\hline 175.9 & 283.0 & & & & & & & & & & & & & & & & & & & 4 \\
\hline 176.5 & 284.0 & & & & & & & & & & 1 & & & & & 2 & 1 & & 2 & \\
\hline 176.7 & 284.3 & & & & & & & & & & & & & & & & & & & 7 \\
\hline 177.9 & 286.2 & & & & & & & & & & & & & & & & & & & 1 \\
\hline 178.3 & 286.9 & & & & & & & & & 1 & & & & & & & 3 & & & 10 \\
\hline 178.5 & 287.2 & & & & & & & & & & & & & & & 2 & & & 38 & 1 \\
\hline 178.9 & 287.9 & & & & 1 & & & & & 1 & & & & 2 & 7 & 13 & & 18 & 10 & 11 \\
\hline 179.5 & 288.8 & & & & & & & & & & & & & & & & & & & 6 \\
\hline 179.6 & 289.0 & & & & & & & & 6 & 13 & 21 & 32 & 5 & 16 & 40 & 56 & 72 & 92 & 110 & 99 \\
\hline 181.7 & 292.4 & & & & & & & & & & & & & & 1 & 1 & 1 & 3 & 9 & 15 \\
\hline 182.3 & 293.3 & & & & & & & & & & & & & & & & & & 8 & \\
\hline 183.1 & 294.6 & & & & & & & & & & & & & & 2 & & & 4 & & 2 \\
\hline 183.4 & 295.1 & & & & & & & & & & & & & & & & & & 1 & 8 \\
\hline 183.5 & 295.3 & & & & & & & & & & & & & & & & & & 11 & 9 \\
\hline 184.7 & 297.2 & & & & & & & & & & & & & & & & & & 6 & 1 \\
\hline 186.7 & 300.4 & & & & & & & & & & & & & & & & & 3 & & \\
\hline 187.5 & 301.7 & & & & & & & & & & & & & & & & 1 & 1 & & \\
\hline 187.7 & 302.0 & & & & & & & & & & & & & & & & 1 & & 7 & 3 \\
\hline 188.2 & 302.8 & & & & & & & & & & & & & & 1 & 2 & 7 & 1 & 3 & 2 \\
\hline 190.0 & 305.7 & & & & & & & & & & 1 & & & & & & & & 1 & 1 \\
\hline 190.1 & 305.9 & & & & & & & & & 1 & & & & & & & 2 & 5 & 3 & \\
\hline 190.2 & 306.0 & & & & & & & & & & & & & & & & & & & 2 \\
\hline 190.7 & 306.8 & & & & & & & & & & & & & & & & & & 3 & \\
\hline 190.8 & 307.0 & & 1 & 5 & & 2 & 5 & 1 & & & & 1 & & 4 & 2 & 5 & 18 & 28 & 6 & 17 \\
\hline 191.0 & 307.3 & & & & & & & & & & & & & & & & & & 2 & 2 \\
\hline
\end{tabular}


Appendix 3 (Continued)

Fall Chinook salmon redds counted in the Snake River during aerial and underwater searches, by river mile (RM), river kilometer (RK), and year (19862004). An empty cell indicates no redds were observed at the corresponding site and year.

\begin{tabular}{|c|c|c|c|c|c|c|c|c|c|c|c|c|c|c|c|c|c|c|c|c|}
\hline \multirow[b]{2}{*}{$\mathrm{RM}$} & \multirow[b]{2}{*}{ RK } & \multicolumn{19}{|c|}{ Year } \\
\hline & & 1986 & 1987 & 1988 & 1989 & 1990 & 1991 & 1992 & 1993 & 1994 & 1995 & 1996 & 1997 & 1998 & 1999 & 2000 & 2001 & 2002 & 2003 & 2004 \\
\hline 191.1 & 307.5 & & & & & & & & & & & & & & & & & & & 1 \\
\hline 191.7 & 308.4 & 2 & 2 & 4 & & & & & & & & & & & 1 & & 3 & 10 & 11 & 6 \\
\hline 193.0 & 310.5 & & & & & & & & & & & & & & & & & 28 & 18 & 22 \\
\hline 193.2 & 310.9 & & & & & & & & & & & & & & & & & & 1 & \\
\hline 193.4 & 311.2 & & & & 5 & 2 & & & & 2 & & 2 & 1 & 4 & & & & 5 & 3 & 1 \\
\hline 193.6 & 311.5 & & & & & & & & & & & & & & & & 6 & 1 & 9 & 6 \\
\hline 193.7 & 311.7 & & 4 & & & & & 6 & 1 & 2 & 1 & & 2 & 3 & & 5 & 8 & 8 & & 11 \\
\hline 193.8 & 311.8 & & & & & & & & 1 & 1 & 1 & & & & 1 & 2 & 1 & 1 & & 3 \\
\hline 194.0 & 312.1 & & 2 & & & 3 & & & 1 & 2 & 4 & 2 & 6 & 14 & 11 & 11 & 22 & 26 & 20 & 21 \\
\hline 194.1 & 312.3 & & & 2 & & 2 & & & & 5 & & & & & & & 1 & & 1 & 6 \\
\hline 194.4 & 312.8 & & & & & & & & & & & & & & & & & 1 & 5 & 23 \\
\hline 196.0 & 315.4 & & & 3 & & & & & & 2 & & & & 1 & 6 & 10 & 20 & 23 & 34 & 29 \\
\hline 196.2 & 315.7 & & 1 & & & & & & & & & & & & & & & & 25 & \\
\hline 198.2 & 318.9 & & & & & & & & & & & 2 & & 1 & 17 & 14 & 36 & 33 & 13 & 35 \\
\hline 198.8 & 319.9 & & 5 & & 3 & 2 & 7 & 3 & & 6 & 1 & 6 & & 4 & 15 & 17 & 21 & 25 & 39 & 43 \\
\hline 199.4 & 320.8 & & & & & & & & 1 & & & 5 & & & 2 & 1 & 2 & & & 1 \\
\hline 201.0 & 323.4 & & & & & & & & & & & & & & & & & & & 4 \\
\hline 201.1 & 323.6 & & & & & & & & & & & & & & 1 & 1 & 2 & 3 & 6 & \\
\hline 203.1 & 326.8 & & & & & & & & & & & & & 10 & & & & 1 & 10 & \\
\hline 204.1 & 328.4 & & 1 & & & & & & & & & & & & & & & & & \\
\hline 205.3 & 330.3 & & & & & & 3 & & & & 1 & & & 3 & 6 & 2 & & 11 & 17 & 12 \\
\hline 205.4 & 330.5 & & 1 & & & & & & & & & 2 & & & & 4 & & 2 & 4 & 5 \\
\hline 205.7 & 331.0 & & & & & & & & & & & & & & & & & & 2 & \\
\hline 206.4 & 332.1 & & 1 & 4 & & & 1 & 2 & 1 & & 2 & & & 2 & 4 & & 2 & 2 & 10 & 15 \\
\hline 206.6 & 332.4 & & & & & & & & & & & & & & 2 & 1 & & 1 & 5 & 2 \\
\hline 207.5 & 333.9 & & & & & & & & & & & & & & & & & & & 1 \\
\hline 207.7 & 334.2 & & & & & & & & & & & & & & 2 & & & 3 & 3 & \\
\hline 207.8 & 334.4 & & & 1 & & & & & & & & 3 & 2 & & 5 & & & 1 & 3 & 4 \\
\hline
\end{tabular}


Appendix 3 (Continued)

Fall Chinook salmon redds counted in the Snake River during aerial and underwater searches, by river mile (RM), river kilometer (RK), and year (19862004). An empty cell indicates no redds were observed at the corresponding site and year.

\begin{tabular}{|c|c|c|c|c|c|c|c|c|c|c|c|c|c|c|c|c|c|c|c|c|}
\hline \multirow[b]{2}{*}{$\mathrm{RM}$} & \multirow[b]{2}{*}{ RK } & \multicolumn{19}{|c|}{ Year } \\
\hline & & 1986 & 1987 & 1988 & 1989 & 1990 & 1991 & 1992 & 1993 & 1994 & 1995 & 1996 & 1997 & 1998 & 1999 & 2000 & 2001 & 2002 & 2003 & 2004 \\
\hline 207.9 & 334.5 & & & 2 & & & & & & & & & & & & & & & & \\
\hline 208.0 & 334.7 & & & & 1 & & & & & & 2 & 9 & 5 & 13 & 36 & 17 & 26 & 37 & 62 & 51 \\
\hline 208.1 & 334.8 & & & & & & & & & & & & & & & & & 1 & & \\
\hline 208.3 & 335.2 & & & & & & & & & & & & & & 4 & & & & & \\
\hline 209.1 & 336.4 & & & & & & & & & & & & & & 1 & & & 1 & & \\
\hline 209.7 & 337.4 & & 1 & & & & & & & & & & & & 1 & & & 2 & & \\
\hline 210.8 & 339.2 & & & & & & & & & & & & & & & & & & 1 & \\
\hline 211.9 & 340.9 & & & & & & & & 2 & & & & & 11 & 10 & 6 & 14 & 25 & 30 & 28 \\
\hline 212.2 & 341.4 & & & & & & & & & & 2 & & & 17 & 24 & 28 & 37 & 37 & 34 & 27 \\
\hline 212.3 & 341.6 & & & & & & & & & & & & & & 2 & 3 & 9 & 9 & 4 & 12 \\
\hline 213.3 & 343.2 & & & & 2 & & & & & & & 1 & & & & 1 & 6 & 9 & 12 & 6 \\
\hline 213.5 & 343.5 & & & & & & & & & & & 1 & & & 2 & 1 & 2 & 4 & 6 & 5 \\
\hline 213.7 & 343.8 & & & & & 1 & & 2 & & & & & & 4 & 1 & 1 & 6 & 7 & 1 & 13 \\
\hline 213.9 & 344.2 & & & & & & & & & & & & & & & & & & & 2 \\
\hline 214.5 & 345.1 & & & & & & & & 1 & & & & & & & & 1 & 4 & 3 & 7 \\
\hline 214.7 & 345.5 & & 2 & & & & & & & & & & & & & & & & & \\
\hline 215.4 & 346.6 & & & & & & & & & & & & & 1 & 2 & & & & 3 & 3 \\
\hline 216.1 & 347.7 & & & & & & & & & 1 & & & & 3 & 1 & 2 & 6 & 4 & 6 & 9 \\
\hline 216.9 & 349.0 & & & & & & & & & & & & & & 4 & 5 & 7 & 21 & 23 & 53 \\
\hline 217.3 & 349.6 & & & & & & & 1 & 3 & & 1 & & & 4 & 24 & 6 & 13 & 22 & 42 & 36 \\
\hline 217.8 & 350.4 & & & & 1 & & & & & & & & & & & & & & 2 & \\
\hline 218.2 & 351.1 & & 1 & & & & & & & & & & & & & & & & & \\
\hline 218.5 & 351.6 & & & & & & & & & & & & & & 3 & 3 & & 16 & 11 & 47 \\
\hline 218.6 & 351.7 & & & & & & & & & & & & & & 1 & & & & & \\
\hline 218.7 & 351.9 & & & & & & & & & & 4 & 7 & & 4 & 12 & 5 & 15 & 17 & 20 & 16 \\
\hline 219.0 & 352.4 & & & & & & & & & & 3 & 2 & & 4 & 5 & 6 & 3 & 11 & 7 & 12 \\
\hline 219.3 & 352.9 & & & 2 & & & & 1 & & 3 & & 2 & 3 & 6 & 8 & 5 & 7 & 13 & 12 & 17 \\
\hline 220.7 & 355.1 & & & & & & & & & & & & & & & & & & 4 & 1 \\
\hline
\end{tabular}


Appendix 3 (Continued)

Fall Chinook salmon redds counted in the Snake River during aerial and underwater searches, by river mile (RM), river kilometer (RK), and year (19862004). An empty cell indicates no redds were observed at the corresponding site and year.

\begin{tabular}{|c|c|c|c|c|c|c|c|c|c|c|c|c|c|c|c|c|c|c|c|c|}
\hline \multirow[b]{2}{*}{$\mathrm{RM}$} & \multirow[b]{2}{*}{$\mathrm{RK}$} & \multicolumn{19}{|c|}{ Year } \\
\hline & & 1986 & 1987 & 1988 & 1989 & 1990 & 1991 & 1992 & 1993 & 1994 & 1995 & 1996 & 1997 & 1998 & 1999 & 2000 & 2001 & 2002 & 2003 & 2004 \\
\hline 221.0 & 355.6 & & & & & & & & & & & & & & & & & 1 & & \\
\hline 221.5 & 356.4 & & & & & & & & & & & & & & & & 1 & & & \\
\hline 222.3 & 357.7 & & & & & & & & & & & & & & & & & 2 & & 6 \\
\hline 222.7 & 358.3 & & & & 1 & & & & & & & & & & 6 & & 1 & & 8 & 14 \\
\hline 222.8 & 358.5 & 2 & 3 & & & & & & & & & & & & & 5 & & & & 2 \\
\hline 222.9 & 358.6 & & & & 3 & & & & 3 & 1 & & & & & 9 & & 4 & 20 & 19 & 21 \\
\hline 223.2 & 359.1 & & & & & & & & & 3 & 3 & & & & 3 & & & 1 & & \\
\hline 223.7 & 359.9 & & & 1 & & & & & & & & & & & & & & & & \\
\hline 224.7 & 361.5 & & & & & & & & & & & & & 3 & & & & & 12 & 15 \\
\hline 225.0 & 362.0 & & & & & & & & & & & & & & 2 & & & 2 & & 1 \\
\hline 225.1 & 362.2 & & & & & & & & & & & & & & 3 & & & 1 & 3 & \\
\hline 226.7 & 364.8 & & & & & & & & & & & & & & 2 & & & & & \\
\hline 228.0 & 366.9 & & & & & & & & & & & & & & 2 & & & & 1 & \\
\hline 228.7 & 368.0 & & & & & & & & & & & & & & & & & & & 3 \\
\hline 231.3 & 372.2 & & & & & & & & & & & & & & & & & 1 & 1 & 2 \\
\hline 235.0 & 378.1 & & & & & & & & & & & & & & & & & & & 57 \\
\hline 235.1 & 378.3 & & & & & & & & & & 1 & & & 1 & 2 & 3 & 8 & 21 & 66 & \\
\hline 235.7 & 379.2 & & 4 & & 3 & & & & & 5 & 2 & 7 & 1 & 4 & 11 & 16 & 16 & 22 & 16 & 25 \\
\hline 236.0 & 379.7 & 1 & 1 & 2 & 1 & & & & & & & & & & 2 & 1 & & & 2 & 3 \\
\hline 236.1 & 379.9 & & & & & & & & & & & & & & & 1 & 1 & 1 & & 2 \\
\hline 236.3 & 380.2 & & & & & & & & & & & & & & & & & & & 1 \\
\hline 236.7 & 380.9 & & 1 & & & & & & & & & & & & & & & & & \\
\hline 237.0 & 381.3 & & & & & & & & 5 & 3 & 1 & & 2 & 8 & 6 & 13 & 14 & 26 & 20 & 26 \\
\hline 238.1 & 383.1 & & & & & & & & & & & & & & & & & & & 1 \\
\hline 238.3 & 383.4 & & & & & & & & & & & & & & 1 & 1 & & 2 & 1 & \\
\hline 238.6 & 383.9 & & 2 & & 2 & & & & & & 1 & & & & 4 & 4 & 8 & 13 & 23 & 16 \\
\hline 239.4 & 385.2 & & & & & & & & & & & & & & & & & & 6 & \\
\hline 240.4 & 386.8 & & & & & & & & & & & & & & & & & & & 15 \\
\hline
\end{tabular}


Appendix 3 (Continued)

Fall Chinook salmon redds counted in the Snake River during aerial and underwater searches, by river mile (RM), river kilometer (RK), and year (19862004). An empty cell indicates no redds were observed at the corresponding site and year.

\begin{tabular}{|c|c|c|c|c|c|c|c|c|c|c|c|c|c|c|c|c|c|c|c|c|}
\hline \multirow[b]{2}{*}{$\mathrm{RM}$} & \multirow[b]{2}{*}{ RK } & \multicolumn{19}{|c|}{ Year } \\
\hline & & 1986 & 1987 & 1988 & 1989 & 1990 & 1991 & 1992 & 1993 & 1994 & 1995 & 1996 & 1997 & 1998 & 1999 & 2000 & 2001 & 2002 & 2003 & 2004 \\
\hline 240.5 & 387.0 & & 6 & & & & & & & & & 1 & & 2 & 8 & 1 & 3 & 11 & 11 & 36 \\
\hline 240.7 & 387.3 & & & & 3 & & 6 & & & & & 1 & 1 & 4 & 7 & 11 & 13 & 21 & 24 & \\
\hline 241.0 & 387.8 & & & & & & & & & & & & 1 & & & & 4 & 5 & 8 & 8 \\
\hline 242.8 & 390.7 & & & & & & & & & & & & & 1 & & 4 & 4 & 9 & 13 & 21 \\
\hline 243.0 & 391.0 & & & & & & & & & & & & & & 2 & & & & & \\
\hline 243.3 & 391.5 & & 1 & & 1 & & & & & & & & & & & 4 & & & & \\
\hline 243.5 & 391.8 & & & & & & & & & & & 2 & & 1 & & & 5 & & 13 & 12 \\
\hline 243.8 & 392.3 & & & & & & & & & & & & & & & & & & 2 & 2 \\
\hline 244.0 & 392.6 & & & & & & & & & & & & & & 2 & & & 12 & & 8 \\
\hline 244.3 & 393.1 & & & & & & & & & & & & & & & & & 2 & & \\
\hline 244.5 & 393.4 & & & & & & & & & & & & & & & & & & & 25 \\
\hline 244.6 & 393.6 & & & & 1 & 2 & & & & & & & & & 1 & 2 & 9 & 13 & 31 & \\
\hline 244.7 & 393.7 & & & & & & & & & & & & & & & & & & 2 & 1 \\
\hline 245.2 & 394.5 & & & & & & & & & & & & & & & & & & & 1 \\
\hline 245.3 & 394.7 & & & & & & & & & & & & & & 1 & & 1 & & 9 & 7 \\
\hline 245.7 & 395.3 & & & & & & & & & & 2 & & & & & & & & 2 & 6 \\
\hline 245.8 & 395.5 & & & & & & & & & & & & & & 2 & & 1 & 4 & 6 & 9 \\
\hline 246.5 & 396.6 & & & 1 & & & & & & & & & & & & & & & & \\
\hline \multirow[t]{2}{*}{247.5} & 398.2 & & & & & & & & & & & & & & & & & & 4 & 2 \\
\hline & & 7 & 66 & 64 & 58 & 37 & 51 & 47 & 127 & 67 & 65 & 104 & 58 & 185 & 373 & 346 & 709 & 1,113 & 1,512 & 1,709 \\
\hline
\end{tabular}




\section{Appendix 4}

Fall Chinook salmon redds counted in the Clearwater River during aerial searches, by river mile (RM), river kilometer (RK), and year (1988-2004). An empty cell indicates no redds were observed at the corresponding site and year. The maximum upstream RM searched was 41 (North Fork Clearwater River) from 1988 to 1990, 67 (Kamiah, Idaho) in 1991, and 74 (beginning of Middle Fork Clearwater River) from 1992 to 2004.

\begin{tabular}{|c|c|c|c|c|c|c|c|c|c|c|c|c|c|c|c|c|c|c|}
\hline \multirow[b]{2}{*}{$\mathrm{RM}$} & \multirow[b]{2}{*}{ RK } & \multicolumn{17}{|c|}{ Year } \\
\hline & & 1988 & 1989 & 1990 & 1991 & 1992 & 1993 & 1994 & 1995 & 1996 & 1997 & 1998 & 1999 & 2000 & 2001 & 2002 & 2003 & 2004 \\
\hline 4.0 & 6.4 & & 1 & & & & & & 1 & & & & & & & & & \\
\hline 6.2 & 10.0 & & & & & & & & & 5 & & & & & & & & \\
\hline 6.7 & 10.8 & & & & & & & & & & & & & & 2 & & & \\
\hline 7.2 & 11.6 & & & & & & & & & & & & & & 2 & & & \\
\hline 7.8 & 12.6 & & & & & & & & & & & & & & 7 & 6 & & \\
\hline 8.0 & 12.8 & & & & & & & & & & & & 1 & & & & & \\
\hline 8.1 & 13.0 & 9 & & & & 1 & 2 & 6 & 6 & & & 1 & & & & & & \\
\hline 8.8 & 14.2 & & & & & & & & & & & & & & 1 & 5 & & \\
\hline 10.6 & 17.1 & & & 1 & & & & & & & & & & & & & & \\
\hline 11.8 & 19.0 & & & & & & 1 & & & & & & & & & & & \\
\hline 13.9 & 22.4 & & 4 & 3 & & & & & & & 1 & & 2 & 8 & 12 & & & 7 \\
\hline 16.2 & 26.1 & & & & & & & & & & & & & & & 1 & & \\
\hline 17.4 & 28.0 & & & & & & & & & & 1 & 2 & 1 & & & 2 & & \\
\hline 18.0 & 29.0 & 4 & 3 & & 1 & & & & & & 1 & 2 & 15 & 2 & 18 & 22 & 36 & 45 \\
\hline 18.6 & 29.9 & & & & & & & & & & 1 & & & & & & 6 & \\
\hline 18.9 & 30.4 & & & & & & & & & & & & 1 & & & & & \\
\hline 19.0 & 30.6 & & & & & 2 & & & & & & & & & & & & \\
\hline 19.1 & 30.7 & & & & & & & & & & & & 3 & & 6 & 17 & 8 & 7 \\
\hline 19.4 & 31.2 & & & & & & & & & & 1 & 4 & 5 & & & & & 31 \\
\hline 19.5 & 31.4 & & & & & & & & & & & & & 7 & 14 & 1 & 11 & 1 \\
\hline 20.0 & 32.2 & & 2 & & & & & & 4 & 6 & 1 & & & & & & & \\
\hline 21.7 & 34.9 & & & & & & & & & & & & & & 2 & 1 & 7 & 3 \\
\hline 21.8 & 35.0 & & & & & & & & & & & & 1 & & & & & \\
\hline 22.0 & 35.4 & 8 & & & & 21 & 9 & 18 & 5 & 24 & 16 & 25 & 62 & 77 & 60 & 110 & 107 & 119 \\
\hline 22.2 & 35.7 & & & & & & & & & & & & & & & & 49 & 22 \\
\hline 23.0 & 37.0 & & & & 3 & & & & & & & & & & 29 & & & \\
\hline 23.3 & 37.5 & & & & & & & & & & & & & & & 9 & 3 & 18 \\
\hline 23.4 & 37.7 & & & & & & & & & & 1 & & & & & & & \\
\hline 24.0 & 38.6 & & & & & & & & & & & & & & 1 & & & 7 \\
\hline 25.5 & 41.0 & & & & & & & & & & & & & & & 1 & 5 & \\
\hline 26.3 & 42.3 & & & & & & & & & & & & & & & & 2 & \\
\hline
\end{tabular}




\section{Appendix 4 (Continued)}

Fall Chinook salmon redds counted in the Clearwater River during aerial searches, by river mile (RM), river kilometer (RK), and year (1988-2004). An empty cell indicates no redds were observed at the corresponding site and year. The maximum upstream RM searched was 41 (North Fork Clearwater River) from 1988 to 1990, 67 (Kamiah, Idaho) in 1991, and 74 (beginning of Middle Fork Clearwater River) from 1992 to 2004.

\begin{tabular}{|c|c|c|c|c|c|c|c|c|c|c|c|c|c|c|c|c|c|c|}
\hline \multirow[b]{2}{*}{$\mathrm{RM}$} & \multirow[b]{2}{*}{$\mathrm{RK}$} & \multicolumn{17}{|c|}{ Year } \\
\hline & & 1988 & 1989 & 1990 & 1991 & 1992 & 1993 & 1994 & 1995 & 1996 & 1997 & 1998 & 1999 & 2000 & 2001 & 2002 & 2003 & 2004 \\
\hline 26.5 & 42.6 & & & & & & & & & & & & & 3 & 2 & 20 & 4 & 18 \\
\hline 26.8 & 43.1 & & & & & & & & & & & 1 & & & & & & \\
\hline 27.6 & 44.4 & & & & & & & & & & & 2 & & & & 15 & 7 & 6 \\
\hline 28.0 & 45.1 & & & & & & & 1 & & & & & & & & 48 & 20 & \\
\hline 28.4 & 45.7 & & & & & & & & & & 11 & 1 & 26 & 1 & & & & 20 \\
\hline 30.1 & 48.4 & & & & & & & & & & & & & & & 3 & 5 & 35 \\
\hline 31.5 & 50.7 & & & & & & & & & & & & & & 3 & 28 & 3 & 37 \\
\hline 31.7 & 51.0 & & & & & & & & & 4 & & 6 & & & & & 37 & \\
\hline 32.5 & 52.3 & & & & & & & & & & & & 23 & 13 & 19 & 11 & & 20 \\
\hline 32.8 & 52.8 & & & & & & & & & & & & & & & 4 & & \\
\hline 33.6 & 54.0 & & & & & & 13 & & & & & & & & & & & \\
\hline 33.8 & 54.4 & & & & & & & & & & & & & 6 & & & & \\
\hline 34.0 & 54.7 & & & & & & & & & & 9 & 4 & 9 & 2 & 13 & 42 & 20 & 15 \\
\hline 34.2 & 55.0 & & & & & & & & & 10 & & & & & & & & \\
\hline 35.0 & 56.3 & & & & & & & & 3 & 6 & 7 & & & & & & & \\
\hline 35.4 & 56.9 & & & & & & & & & & & & 2 & 9 & 47 & 41 & 53 & 50 \\
\hline 35.7 & 57.5 & & & & & & & & & & & & 3 & & 1 & 7 & 7 & \\
\hline 36.2 & 58.2 & & & & & & & & & & 6 & 11 & 3 & 11 & & 7 & 4 & 10 \\
\hline 36.7 & 59.0 & & & & & & & 1 & & & & & & & & & & \\
\hline 37.9 & 61.0 & & & & & & & & & & & & & & 1 & 8 & 1 & 1 \\
\hline 39.1 & 62.9 & & & & & & & & & & & & & & & & 1 & 9 \\
\hline 39.5 & 63.6 & & & & & & & & & & 1 & 9 & 1 & & 4 & 2 & 1 & \\
\hline 40.3 & 64.8 & & & & & 1 & 11 & 4 & 1 & 11 & 1 & 10 & 21 & 22 & 46 & 109 & 147 & 111 \\
\hline 40.6 & 65.3 & & & & & & & & & & & & & 3 & & & & \\
\hline 43.2 & 69.5 & & & & & & & & & & & & & & & 1 & 1 & \\
\hline 45.0 & 72.4 & & & & & & & & & & & & & 3 & 9 & 2 & 2 & 10 \\
\hline 49.2 & 79.2 & & & & & & & & & & & & & 1 & & & & 3 \\
\hline 49.4 & 79.5 & & & & & & & & & & & & & & & & 1 & \\
\hline 49.8 & 80.1 & & & & & & & & & & & & & & & & & 1 \\
\hline 51.2 & 82.4 & & & & & & & & & & & & & & & & & 3 \\
\hline 51.5 & 82.9 & & & & & & & & & & & & & & & & & 5 \\
\hline
\end{tabular}




\section{Appendix 4 (Continued)}

Fall Chinook salmon redds counted in the Clearwater River during aerial searches, by river mile (RM), river kilometer (RK), and year (1988-2004). An empty cell indicates no redds were observed at the corresponding site and year. The maximum upstream RM searched was 41 (North Fork Clearwater River) from 1988 to 1990, 67 (Kamiah, Idaho) in 1991, and 74 (beginning of Middle Fork Clearwater River) from 1992 to 2004.

\begin{tabular}{|c|c|c|c|c|c|c|c|c|c|c|c|c|c|c|c|c|c|c|}
\hline \multirow[b]{2}{*}{$\mathrm{RM}$} & \multirow[b]{2}{*}{ RK } & \multicolumn{17}{|c|}{ Year } \\
\hline & & 1988 & 1989 & 1990 & 1991 & 1992 & 1993 & 1994 & 1995 & 1996 & 1997 & 1998 & 1999 & 2000 & 2001 & 2002 & 2003 & 2004 \\
\hline 51.7 & 83.2 & & & & & & & & & & & & & 3 & & & & \\
\hline 52.0 & 83.7 & & & & & 1 & & & & & & & & & 3 & & & \\
\hline 52.8 & 85.0 & & & & & & & & & & & & & & & & 3 & 2 \\
\hline 53.4 & 85.9 & & & & & & & & & & & & 2 & & & & & \\
\hline 53.8 & 86.6 & & & & & & & & & & & & & & & & & 12 \\
\hline 57.2 & 92.0 & & & & & & & & & & & & & & & & 9 & \\
\hline 61.0 & 98.1 & & & & & & & & & & & & & 1 & & & & \\
\hline 62.4 & 100.4 & & & & & & & & & & & & & & & & 1 & \\
\hline 66.0 & 106.2 & & & & & & & & & & & & & & 4 & 1 & & \\
\hline 70.2 & 113.0 & & & & & & & & & & & & & & & & 2 & \\
\hline & & 21 & 10 & 4 & 4 & 26 & 36 & 30 & 20 & 66 & 58 & 78 & 181 & 172 & 306 & 524 & 563 & 628 \\
\hline
\end{tabular}




\section{Appendix 5}

Fall Chinook salmon redds counted in the Grande Ronde River during aerial searches, by river mile (RM), river kilometer (RK), and year (1992-2004). An empty cell indicates no redds were observed at the corresponding site and year. Redd searches were also conducted from 1986 to 1991, however, no ground locations were recorded. Redd counts totaled 0 in 1986, 7 in 1987, 1 in 1988, 0 in 1989, 1 in 1990, and 0 in 1991. The maximum upstream RM searched was 4.5 in 1986, 36 in 1987, and 45.5 from 1988 to 1991, 45.3 in 1992, 53 in 1993 and 1994, 45.3 in 1995, and 53 from 1996 to 2004.

\begin{tabular}{|c|c|c|c|c|c|c|c|c|c|c|c|c|c|c|}
\hline \multirow[b]{2}{*}{$\mathrm{RM}$} & \multirow[b]{2}{*}{ RK } & \multicolumn{13}{|c|}{ Year } \\
\hline & & 1992 & 1993 & 1994 & 1995 & 1996 & 1997 & 1998 & 1999 & 2000 & 2001 & 2002 & 2003 & 2004 \\
\hline 0.1 & 0.2 & & & & & & & & & & 12 & & 2 & 1 \\
\hline 0.5 & 0.8 & & & & & & & & & & & & 2 & \\
\hline 0.7 & 1.1 & & & & & & & & & & 22 & & & \\
\hline 0.8 & 1.3 & & & & & & & & & & & 2 & & \\
\hline 1.0 & 1.6 & & & & & & & & & & 2 & & 4 & 2 \\
\hline 1.2 & 1.9 & & & & & & & & & & 2 & & & \\
\hline 1.9 & 3.1 & & & & & & & & & & 3 & 8 & & \\
\hline 2.0 & 3.2 & & & & & & & & & & 2 & & & 30 \\
\hline 2.1 & 3.4 & & 2 & & & & & & & & & & 2 & \\
\hline 2.2 & 3.5 & & & & & & & & & & 4 & & & \\
\hline 2.4 & 3.9 & & & & & & & & & & & & & 2 \\
\hline 3.0 & 4.8 & & & & & & & 1 & & & 1 & 1 & & \\
\hline 3.2 & 5.1 & 1 & 1 & & & & 1 & 1 & & & 7 & 5 & 2 & 3 \\
\hline 3.3 & 5.3 & & & & & & & & & & & & & 1 \\
\hline 3.5 & 5.6 & & & & & & & & & & & & 1 & \\
\hline 3.6 & 5.8 & & & & 2 & 2 & & & & 1 & 14 & 3 & 4 & 31 \\
\hline 4.3 & 6.9 & & & & & & & & & & & & & 4 \\
\hline 4.4 & 7.1 & 2 & 4 & & & & 1 & 3 & 1 & 1 & 1 & 9 & 6 & 2 \\
\hline 4.5 & 7.2 & & & & & & & & & & 2 & 1 & 3 & \\
\hline 4.6 & 7.4 & & 2 & & & 1 & & & & & 7 & & 1 & 1 \\
\hline 5.5 & 8.8 & & & & & & & & & & 8 & & & 1 \\
\hline 6.2 & 10.0 & & & & & & & & & & 4 & & & \\
\hline 6.5 & 10.5 & & & & & & & & & & & & 1 & \\
\hline 6.8 & 10.9 & & & & & & & & & & 1 & & & \\
\hline 7.9 & 12.7 & & & & & & & & & & 1 & & & \\
\hline 8.2 & 13.2 & & & & & & & & & & & 1 & 1 & \\
\hline 8.5 & 13.7 & & & & & & & & & & 7 & & & \\
\hline 8.9 & 14.3 & & & & & & & & & & & & 1 & \\
\hline 9.2 & 14.8 & & & & & & & & & & 6 & & & 1 \\
\hline 9.6 & 15.4 & & & & & & 1 & & & & & & & \\
\hline 10.0 & 16.1 & & & & & & & & & & & 2 & & 3 \\
\hline 10.4 & 16.7 & & & & & & & & & & & 6 & & \\
\hline 10.5 & 16.9 & & 5 & 1 & & 6 & 7 & 2 & & & 9 & 6 & & 13 \\
\hline 11.0 & 17.7 & & & & & & & & & & & & 1 & \\
\hline 11.4 & 18.3 & & & & & & & & & & & & & 1 \\
\hline 11.6 & 18.7 & & 2 & & & & & & & & & 1 & & \\
\hline 11.7 & 18.8 & & & & & & & & & & 3 & & 2 & 2 \\
\hline 12.0 & 19.3 & & & & & & & & & & 2 & & & \\
\hline 12.5 & 20.1 & & & & & & & & & & 12 & 4 & 4 & 3 \\
\hline 12.6 & 20.3 & & 2 & & & & 6 & 5 & & & 6 & & & 2 \\
\hline
\end{tabular}




\section{Appendix 5 (Continued)}

Fall Chinook salmon redds counted in the Grande Ronde River during aerial searches, by river mile (RM), river kilometer (RK), and year (1992-2004). An empty cell indicates no redds were observed at the site in the corresponding year. Redd searches were also conducted from 1986 to 1991, however, no ground locations were recorded. Redd counts totaled 0 in 1986, 7 in 1987, 1 in 1988, 0 in 1989, 1 in 1990, and 0 in 1991. Redd counts totaled 0 in 1986, 7 in 1987, 1 in 1988, 0 in 1989, 1 in 1990, and 0 in 1991. The maximum upstream RM searched was 4.5 in 1986, 36 in 1987, and 45.5 from 1988 to 1991, 45.3 in 1992, 53 in 1993 and 1994, 45.3 in 1995, and 53 from 1996 to 2004.

\begin{tabular}{|c|c|c|c|c|c|c|c|c|c|c|c|c|c|c|}
\hline \multirow[b]{2}{*}{$\mathrm{RM}$} & \multirow[b]{2}{*}{$\mathrm{RK}$} & \multicolumn{13}{|c|}{ Year } \\
\hline & & 1992 & 1993 & 1994 & 1995 & 1996 & 1997 & 1998 & 1999 & 2000 & 2001 & 2002 & 2003 & 2004 \\
\hline 12.7 & 20.4 & & 2 & & & & & & & & 3 & & & \\
\hline 13.2 & 21.2 & & 3 & & & 1 & & & & & & & & \\
\hline 13.4 & 21.6 & & & & & & & & & & & & & 3 \\
\hline 13.8 & 22.2 & & 7 & & 4 & & & & & & 1 & 2 & 3 & 8 \\
\hline 13.9 & 22.4 & & & & & & & & & & & 2 & & \\
\hline 14.2 & 22.8 & & & & & & & & & & & & & 2 \\
\hline 14.3 & 23.0 & & & & & & & & & & & & & 4 \\
\hline 15.0 & 24.1 & & & & & & & & & & & & 1 & \\
\hline 16.3 & 26.2 & & & & & & & & & & & & 1 & 2 \\
\hline 16.8 & 27.0 & & & & & & & & & & & 1 & & \\
\hline 17.0 & 27.4 & & & 1 & & & & & & & & & & \\
\hline 17.6 & 28.3 & & 2 & 3 & 5 & & 9 & & & & 10 & 13 & 5 & 6 \\
\hline 18.0 & 29.0 & & & & & & 4 & & & & & & 6 & \\
\hline 18.6 & 29.9 & 1 & & & & & & & & & & & & \\
\hline 19.1 & 30.7 & & & & & & & & & & 1 & 3 & & \\
\hline 19.2 & 30.9 & & & & & & 3 & & & & & 2 & 2 & \\
\hline 19.5 & 31.4 & & & & & & & 2 & & & & & & \\
\hline 20.0 & 32.2 & & & & & & 4 & 5 & & & 2 & & 1 & \\
\hline 21.0 & 33.8 & & & & & & & & & & 3 & & 1 & \\
\hline 21.5 & 34.6 & & & & & & & & & & & & 2 & \\
\hline 22.0 & 35.4 & & & & & & & & & & 1 & & & \\
\hline 22.1 & 35.6 & & & & & & & & & & & & & 1 \\
\hline 22.2 & 35.7 & & & & & & & & & & & & & 1 \\
\hline 24.0 & 38.6 & & & & & & & & & & & & & 2 \\
\hline 24.4 & 39.3 & & & & & & & & & & & & & 1 \\
\hline 25.6 & 41.2 & & 4 & & & & & & & & & & & \\
\hline 26.4 & 42.5 & & & 7 & & & & & & & 1 & & 1 & \\
\hline 26.7 & 43.0 & & & & & & & & & & & & & 1 \\
\hline 26.9 & 43.3 & & & & & & & & & & & & 5 & \\
\hline 27.1 & 43.6 & & & & & & & & & & 2 & & & \\
\hline 27.3 & 43.9 & & & & & & & & & & 2 & & 2 & \\
\hline 27.9 & 44.9 & & & & & 3 & & & 6 & & & & & 1 \\
\hline 29.1 & 46.8 & & & & & & & & & & & 2 & 1 & 5 \\
\hline 29.3 & 47.1 & & & & & & & & & & & 1 & & \\
\hline 29.7 & 47.8 & & & & & & & & & & & 3 & & \\
\hline 29.8 & 47.9 & & & & & & & & & & & 1 & & \\
\hline 31.1 & 50.0 & & & & & & & & & & & 3 & & 1 \\
\hline 32.2 & 51.8 & & & 1 & & & 1 & & & & & & & \\
\hline
\end{tabular}




\section{Appendix 5 (Continued)}

Fall Chinook salmon redds counted in the Grande Ronde River during aerial searches, by river mile (RM), river kilometer (RK), and year (1992-2004). An empty cell indicates no redds were observed at the site in the corresponding year. Redd searches were also conducted from 1986 to 1991, however, no ground locations were recorded. Redd counts totaled 0 in 1986, 7 in 1987, 1 in 1988, 0 in 1989, 1 in 1990, and 0 in 1991. Redd counts totaled 0 in 1986, 7 in 1987, 1 in 1988, 0 in 1989, 1 in 1990, and 0 in 1991. The maximum upstream RM searched was 4.5 in 1986, 36 in 1987, and 45.5 from 1988 to 1991, 45.3 in 1992, 53 in 1993 and 1994, 45.3 in 1995, and 53 from 1996 to 2004.

\begin{tabular}{|c|c|c|c|c|c|c|c|c|c|c|c|c|c|c|}
\hline \multirow[b]{2}{*}{$\mathrm{RM}$} & \multirow[b]{2}{*}{$\mathrm{RK}$} & \multicolumn{13}{|c|}{ Year } \\
\hline & & 1992 & 1993 & 1994 & 1995 & 1996 & 1997 & 1998 & 1999 & 2000 & 2001 & 2002 & 2003 & 2004 \\
\hline 32.7 & 52.6 & & & & & & 4 & & & & & & & \\
\hline 33.1 & 53.3 & & & & & & & & & & & 1 & & \\
\hline 33.3 & 53.6 & 1 & & 1 & & 1 & 2 & 3 & & & 10 & 7 & 3 & \\
\hline 34.0 & 54.7 & & 2 & & & & & & & & & & & \\
\hline 36.4 & 58.6 & & & & & & 1 & & & & & & & \\
\hline 37.5 & 60.3 & & & & & & & & & & 2 & & & \\
\hline 37.6 & 60.5 & & 2 & & 1 & & & 2 & & 2 & 2 & 4 & 4 & 4 \\
\hline 37.8 & 60.8 & & 7 & 1 & & & 3 & & & & & & 2 & \\
\hline 39.0 & 62.8 & & & & & & & & & & 3 & & & \\
\hline 39.1 & 62.9 & & & & & & 1 & & & & & & & \\
\hline 41.3 & 66.5 & & & & & & 2 & & & & & & & \\
\hline 41.7 & 67.1 & & 1 & & & & & & & & & & & \\
\hline 41.8 & 67.3 & & & & & 1 & & & & & & & & \\
\hline 42.5 & 68.4 & & & & & & & & & & & & 1 & \\
\hline 43.2 & 69.5 & & 1 & & 3 & & & & 2 & & 11 & 8 & 1 & 1 \\
\hline 44.5 & 71.6 & & & & & & & & & & & 2 & & 4 \\
\hline 44.9 & 72.2 & & & & & & & & & 4 & & 2 & 4 & 5 \\
\hline 45.7 & 73.5 & & & & & & & & & & & 4 & & 4 \\
\hline 45.9 & 73.9 & & & & & 4 & & & & & & & & \\
\hline 46.5 & 74.8 & & & & 2 & & & & & & & & & \\
\hline 47.5 & 76.4 & & & & & & 1 & & 4 & & & & & \\
\hline 47.8 & 76.9 & & & & & & & & & & 2 & & 10 & \\
\hline 48.7 & 78.4 & & & & & & & & & & & & & 2 \\
\hline 49.3 & 79.3 & & & & & & & & & & 3 & 1 & & 1 \\
\hline 50.5 & 81.3 & & & & & & 4 & & & & & & & \\
\hline \multirow[t]{2}{*}{51.5} & 82.9 & & & & 1 & 1 & & & & & & & & \\
\hline & & 5 & 49 & 15 & 18 & 20 & 55 & 24 & 13 & 8 & 197 & 111 & 93 & 162 \\
\hline
\end{tabular}




\section{Appendix 6}

Fall Chinook salmon redds counted in the Salmon River during aerial searches, by river mile (RM), river kilometer (RK), and year (1992-2004). An empty cell indicates no redds were observed at the corresponding site and year. The maximum upstream RM searched was 87 in 1992, 97 in 1993, 134 in 1994, 105 in 1995, 87 in 1996, 134 in 1997, 105 in 1998, 96 in 1999 and 2000, and 105 from 2001 to 2004.

\begin{tabular}{|c|c|c|c|c|c|c|c|c|c|c|c|c|c|c|}
\hline \multirow[b]{2}{*}{$\mathrm{RM}$} & \multirow[b]{2}{*}{$\mathrm{RK}$} & \multicolumn{13}{|c|}{ Year } \\
\hline & & 1992 & 1993 & 1994 & 1995 & 1996 & 1997 & 1998 & 1999 & 2000 & 2001 & 2002 & 2003 & 2004 \\
\hline 4.0 & 6.4 & & & & & & & & & & & & 1 & 6 \\
\hline 4.8 & 7.7 & & & & & & & & & & 1 & & & 1 \\
\hline 5.5 & 8.8 & & & & & & & & & & & & 1 & \\
\hline 5.6 & 9.0 & & & & & & & & & & & & 1 & \\
\hline 14.2 & 22.8 & & & & & & & & & & 1 & & & \\
\hline 15.0 & 24.1 & 1 & & & & & & 1 & & & & & & \\
\hline 15.3 & 24.6 & & & & & & & & & & 2 & & & \\
\hline 15.7 & 25.3 & & & & & & & & & & 1 & & & \\
\hline 16.0 & 25.7 & & & & 1 & 1 & & & & & & & & \\
\hline 20.3 & 32.7 & & & & & & & & & & & & 1 & \\
\hline 20.6 & 33.1 & & & & & & & & & & & & 2 & \\
\hline 24.4 & 39.3 & & & & & & & & & & & & & 1 \\
\hline 26.1 & 42.0 & & & & 1 & & & & & & & & & \\
\hline 26.5 & 42.6 & & & & & & & & & & 1 & & & \\
\hline 30.9 & 49.7 & & & & & & & & & & & & 5 & 8 \\
\hline 31.1 & 50.0 & & 1 & & & & & 1 & & & & & 1 & 1 \\
\hline 31.4 & 50.5 & & & & & & & & & & 1 & & & \\
\hline 35.0 & 56.3 & & & & & & 1 & & & & & & & \\
\hline 35.7 & 57.4 & & & & & & & & & & & 11 & & 1 \\
\hline 45.2 & 72.7 & & & & & & & & & & & 3 & & \\
\hline 48.8 & 78.5 & & & & & & & & & & 3 & & & \\
\hline 56.7 & 91.2 & & & & & & & & & & 1 & & & \\
\hline 62.0 & 99.8 & & & & & & & & & & 3 & 8 & & \\
\hline 63.9 & 102.8 & & & & & & & & & & & 2 & 2 & \\
\hline 65.0 & 104.6 & & & & & & & & & & 2 & & & \\
\hline 65.4 & 105.2 & & & & & & & 1 & & & & 2 & & \\
\hline 65.7 & 105.7 & & & & & & & & & & 2 & 3 & & \\
\hline 65.8 & 105.9 & & & & & & & & & & 1 & & & \\
\hline 68.5 & 110.2 & & & & & & & & & & & & 2 & \\
\hline 70.5 & 113.4 & & & & & & & & & & 1 & & & \\
\hline 70.6 & 113.6 & & & & & & & & & & 1 & & & \\
\hline 82.2 & 132.3 & & & & & & & & & & & & & 1 \\
\hline 85.0 & 136.8 & & & & & & & & & & 1 & & & \\
\hline 87.0 & 140.0 & & & 1 & & & & & & & & & & \\
\hline 91.0 & 146.4 & & & & & & & & & & & & 1 & \\
\hline 88.0 & 141.6 & & & & & & & & & & & 2 & & \\
\hline 90.7 & 146.0 & & 2 & & & & & & & & & & & \\
\hline 100.7 & 162.0 & & & & & & & & & & & & 1 & \\
\hline \multirow[t]{2}{*}{110.0} & 177.0 & & & & & & & & & & & & & 2 \\
\hline & & 1 & 3 & 1 & 2 & 1 & 1 & 3 & 0 & 0 & 22 & 31 & 18 & 21 \\
\hline
\end{tabular}




\section{Appendix 7}

Fall Chinook salmon redds counted in the Imnaha River during aerial searches, by river mile (RM), river kilometer (RK), and year (1992-2004). An empty cell indicates no redds were observed at the corresponding site and year. A dash indicates no searches were conducted in the corresponding site and year. Redd searches were also conducted from 1987 to 1991, and in 1999, 2001, and 2003, however, no ground locations were recorded. Redd counts totaled 0 in 1987, one in 1988 and 1989, three in 1990, four in 1991, three in 1992, nine in 1999, 38 in 2001, and 43 in 2003. The maximum upstream RM searched was 4.1, 19, 9.8, 3.8, 3.8, 14, 35, 35, and 19 respectively.

\begin{tabular}{|c|c|c|c|c|c|c|c|c|c|c|c|c|c|}
\hline \multirow[b]{2}{*}{ RM } & \multirow[b]{2}{*}{ RK } & \multicolumn{12}{|c|}{ Year } \\
\hline & & 1993 & 1994 & 1995 & 1996 & 1997 & 1998 & ----- & 2000 & ----- & 2002 & ----- & 2004 \\
\hline 0.2 & 0.3 & & & & & & & & & & 3 & & \\
\hline 0.3 & 0.5 & & & & & & & & & & 7 & & \\
\hline 0.4 & 0.6 & & & & & & & & & & 3 & & 3 \\
\hline 0.5 & 0.8 & 1 & & 1 & 2 & 1 & 1 & & 1 & & & & 4 \\
\hline 0.6 & 1.0 & & & & & & & & & & 2 & & \\
\hline 0.6 & 1.0 & 1 & & & 1 & & 2 & & 2 & & & & \\
\hline 0.9 & 1.4 & & & & & & & & & & 2 & & \\
\hline 1.0 & 1.6 & & & & & 2 & 1 & & & & & & 2 \\
\hline 1.2 & 1.9 & & & & & & 1 & & & & & & 1 \\
\hline 1.3 & 2.1 & & & & & & & & & & 1 & & 1 \\
\hline 1.4 & 2.3 & & & & & & 1 & & & & & & \\
\hline 1.5 & 2.4 & & & & & & & & & & 3 & & \\
\hline 1.6 & 2.6 & & & & & & & & & & 3 & & \\
\hline 1.7 & 2.7 & & & & & & & & & & 2 & & \\
\hline 1.8 & 2.9 & & & 2 & & & & & & & 1 & & \\
\hline 2.0 & 3.2 & & & & & & & & & & 2 & & \\
\hline 2.3 & 3.7 & & & & & & & & & & 1 & & \\
\hline 2.4 & 3.9 & & & & & & 2 & & 2 & & 7 & & 2 \\
\hline 2.5 & 4.0 & & & & & & & & & & 9 & & \\
\hline 2.7 & 4.3 & & & & & & & & & & 2 & & 2 \\
\hline 2.8 & 4.5 & & & & & & & & & & & & 1 \\
\hline 2.9 & 4.7 & & & & & & & & & & 1 & & \\
\hline 3.0 & 4.8 & & & & & & & & 2 & & & & \\
\hline 3.4 & 5.5 & & & & & & 1 & & & & 2 & & \\
\hline 3.7 & 6.0 & 2 & & & & & & & & & 1 & & \\
\hline 3.8 & 6.1 & & & & & & & & & & & & 1 \\
\hline 4.1 & 6.6 & & & 1 & & & & & & & 1 & & \\
\hline 4.4 & 7.1 & & & & & & & & & & & & 1 \\
\hline 5.4 & 8.7 & - & - & & - & & & & & & 1 & & \\
\hline 6.0 & 9.7 & - & - & & - & & & & & & & & 1 \\
\hline 6.5 & 10.5 & - & - & & - & & & & 1 & & & & 2 \\
\hline 7.0 & 11.3 & - & - & & - & & & & & & & & 1 \\
\hline
\end{tabular}




\section{Appendix 7 (Continued)}

Fall Chinook salmon redds counted in the Imnaha River during aerial searches, by river mile (RM), river kilometer (RK), and year (1992-2004). An empty cell indicates no redds were observed at the corresponding site and year. A dash indicates no searches were conducted in the corresponding site and year. Redd searches were also conducted from 1987 to 1991, and in 1999, 2001, and 2003, however, no ground locations were recorded. Redd counts totaled 0 in 1987, one in 1988 and 1989, three in 1990, four in 1991, three in 1992, nine in 1999, 38 in 2001, and 43 in 2003. The maximum upstream RM searched was 4.1, 19, 9.8, 3.8, 3.8, 14, 35, 35, and 19 respectively.

\begin{tabular}{|c|c|c|c|c|c|c|c|c|c|c|c|c|c|}
\hline \multirow[b]{2}{*}{$\mathrm{RM}$} & \multirow[b]{2}{*}{$\mathrm{RK}$} & \multicolumn{12}{|c|}{ Year } \\
\hline & & 1993 & 1994 & 1995 & 1996 & 1997 & 1998 & ----- & 2000 & ----- & 2002 & ----- & 2004 \\
\hline 7.1 & 11.4 & - & - & & - & & & & & & 10 & & \\
\hline 7.8 & 12.6 & - & - & & - & & & & & & & & 9 \\
\hline 8.2 & 13.2 & - & - & & - & & & & & & & & 1 \\
\hline 8.9 & 14.3 & - & - & & - & & & & & & & & 1 \\
\hline 9.3 & 15.0 & - & - & & - & & & & & & & & 1 \\
\hline 9.9 & 15.9 & - & - & & - & & & & & & 1 & & \\
\hline 10.0 & 16.1 & - & - & & - & & 2 & & 1 & & & & \\
\hline 12.0 & 19.3 & - & - & & - & & 1 & & & & & & \\
\hline 12.8 & 20.6 & - & - & & - & & & & & & 1 & & \\
\hline 13.0 & 20.9 & - & - & & - & & 1 & & & & 1 & & \\
\hline 13.4 & 21.6 & - & - & & - & & & & & & 2 & & \\
\hline 13.5 & 21.7 & - & - & & - & & & & & & 1 & & \\
\hline 14.3 & 23.0 & - & - & & - & & & & & & & & 1 \\
\hline 18.2 & 29.3 & - & - & & - & & & & & & 1 & & \\
\hline \multirow[t]{2}{*}{20.7} & 33.3 & - & - & & - & & & & & & 1 & & \\
\hline & & 4 & 0 & 4 & 3 & 3 & 13 & & 9 & & 72 & & 35 \\
\hline
\end{tabular}

